\title{
NEURAL MECHANISMS OF CORTICO-CORTICAL INTERACTION IN TEXTURE BOUNDARY DETECTION: A MODELING APPROACH
}

\author{
A. THIELSCHER ${ }^{a *}$ AND H. NEUMANN ${ }^{b}$ \\ ${ }^{a}$ Department of Psychiatry, University of Ulm, Leimgrubenweg 12-14, \\ 89075 Ulm, Germany \\ ${ }^{b}$ Department of Neural Information Processing, University of UIm, UIm, \\ Germany
}

\begin{abstract}
Texture information is an elementary feature utilized by the human visual system to automatically, or preattentively, segment the visual scene. The neural substrate underlying human texture processing as well as the basic computational mechanisms remains largely unknown up to now. We propose a neural model of texture processing which integrates the data obtained by a variety of methods into a common computational framework. It consists of a hierarchy of bi-directionally linked visual areas each containing topographical maps of mutually interconnected cells. It builds upon the two key hypotheses that (i) texture segmentation is based on boundary detection and that (ii) texture border detection is mainly a function of higher visual cortical areas such as V4. This model, while attempting to explain the processing of textures, is embedded in a more general neural model architecture of the infero-temporal pathway of form processing.

The model allows to link human performance in texture segmentation with model cell activation patterns, in turn permitting to trace back fundamental psychophysical results on texture processing to their putative neural origins. Most importantly, it enables us to identify and evaluate the functional role of feedback connections between cortical areas in the context of texture processing, namely the suppression of ambiguous cell activities leading to a sharply localized detection of texture boundaries. One of the likely neural origins of modulatory effects on V1 cell activation levels, as observed in electrophysiological studies using single- and multi-unit recordings, can be resolved. (๖) 2003 IBRO. Published by Elsevier Ltd. All rights reserved.
\end{abstract}

Key words: segmentation, texture processing, computational model, psychophysics, context modulation, feedback connections.

The human visual system reliably detects and identifies objects even in complex and cluttered environments. This capability crucially depends on robust grouping and segmentation of the initial visual stimulus into distinct items, thus enabling the segregation of figural components from the background as well as the adaptive focusing on important parts of the scene (Grossberg, 1980, Kastner and Ungerleider, 2000). Segmentation is an automatic and

${ }^{*}$ Corresponding author. Tel: +49-731-500-21535; fax: +49-731-50026751.

E-mail address: axel.thielscher@medizin.uni-ulm.de (A. Thielscher). Abbreviations: LGN, lateral geniculate nuclei; OC, border orientation contrast. pre-attentive process based on the detection of pattern arrangements that signal coherent or salient surface quantities. A stimulus is processed by the visual system in order to extract a variety of features along several dimensions, such as color, luminance, motion or stereoscopic depth. In particular, the use of texture information for segmentation is compelling, as it requires a sophisticated analysis and comparison of surface properties over wide areas of the visual field. For example, the detection of the Dalmatian in Fig. 1 clearly relies on texture, since no other information can be utilized for object segregation, such as, e.g. different luminance levels or continuous contrast contours. Texture processing has been intensively studied by many authors using psychophysical, physiological and functional imaging methods that have led to a vast amount of experimental data. However, the underlying neural mechanisms of texture segregation are still unknown and a focus of ongoing investigation. In this paper, we present a neural computational model which integrates the experimental data obtained by a variety of experimental methods into a common framework, allowing to trace back the known results on human texture processing to their probable neural origins.

Based on the results of early experiments by Julesz (1965) and Beck (1966) many psychophysical studies have further investigated the features that characterize two juxtaposed textures such that they can be automatically, or pre-attentively, separated from each other (Julesz, 1981; Beck, 1983; Bergen and Adelson, 1988; Landy and Bergen, 1991). Common to all these studies is the finding that orientation and size are key features for the segregation of otherwise homogeneous texture regions. The influence of other features like crossings or terminations on automatic segmentation has been identified to be substantially weaker. Furthermore, psychophysical and physiological studies show that human texture processing is based on the detection of boundaries between otherwise homogeneous texture regions. Segmentation based on feature differences, leading to, e.g. orientation contrast between bar items, was investigated psychophysically in detail by Nothdurft $(1985,1991)$. He demonstrated that the ability to segregate between regions is based on structure gradients and thus depends on the detection of local first-order feature dissimilarities of textures signaling their borders within regions. Having stimuli composed of lines that form continuous flow patterns (see Fig. 6), both a central pop-out area and the background may contain lines of same orientation or statistics of oriented items. The central area nevertheless pops out pre-attentively when the orientation contrast along the border is strong enough, that is the 


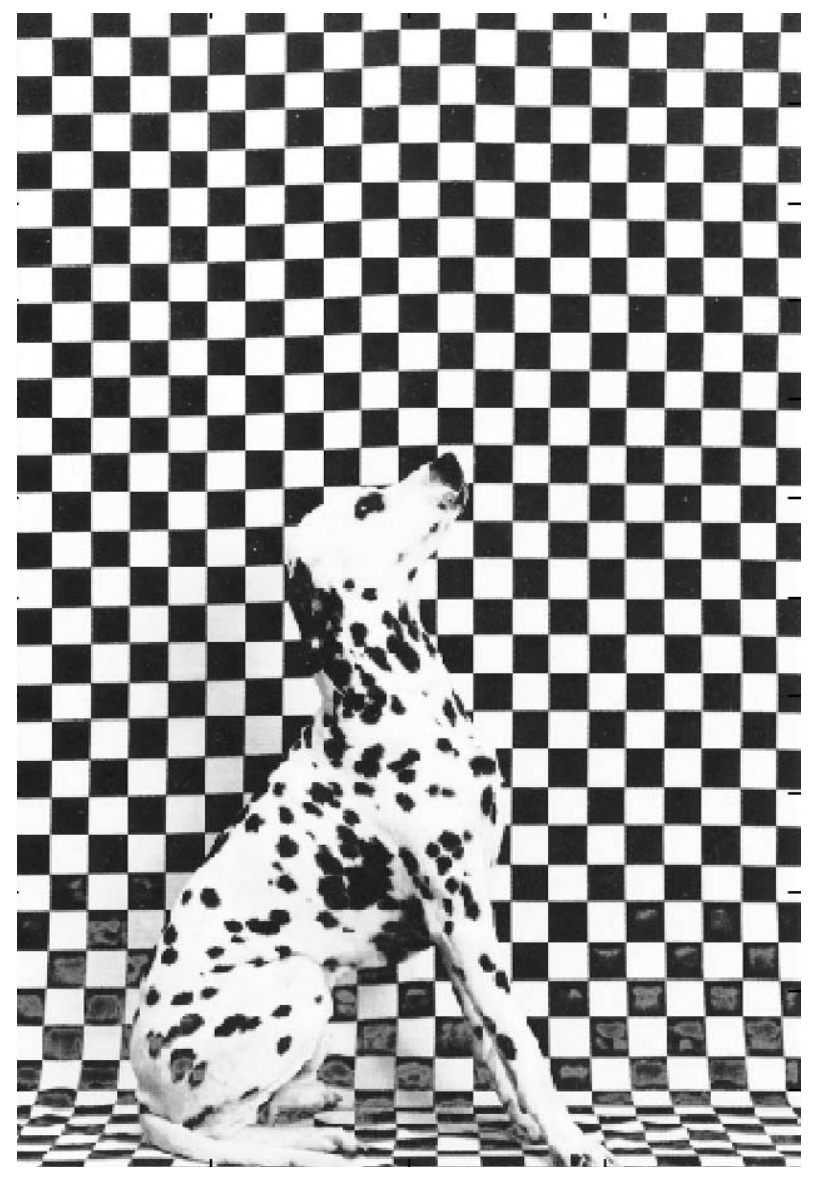

Fig. 1. Example of figure-ground-segmentation relying on texture information. The silhouette of the dog is defined by the change between the regular background texture containing black and white squares and the randomly patterned skin of the dog. (Original figure reprinted with permission by Gert Weigelt 1985/Art Unlimited Amsterdam; image size $400 \times 590)$.

slope in the gradient of texture orientation is sufficiently large. These findings indicate that the visual system does not achieve segmentation by grouping together similar feature elements of a region (according to a homogeneity criterion), but rather by detecting boundaries defined by sufficient contrast along a particular feature dimension. In this case, the robust identification of texture boundaries acts as necessary prerequisite for the reliable reconstruction of region surfaces for figure-ground segmentation. This evidence is further supported by the results of singleand multi-unit recordings in monkey primary visual cortex, which revealed enhanced activity of orientation selective cells when texture borders defined by an orientation contrast fell on their receptive fields (Gallant et al., 1995; Lamme et al., 1998).

Robust segmentation of stimuli embedded in background orientation noise necessitates the pooling of information over extended areas of the retinal image in order to selectively identify meaningful orientation contrasts which signal a texture border. In the visual system, receptive field sizes increase monotonously within the hierarchy of cortical areas (Smith et al., 2001). Recent functional imaging studies demonstrated a significant contribution of higher visual areas, including V4, in texture segmentation as well as in illusory contour detection (Mendola et al., 1999; Kastner et al., 2000). Likewise, lesion studies in monkeys showed that the detection of illusory contours and texture borders as well as the discrimination of shapes is impaired after ablation of V4 (de Weerd et al., 1996; Merigan, 1996, 2000). By this, cells in higher visual areas, such as V4, are likely to provide the necessary substrate of detecting texture boundaries in complex and noisy scenes.

Taken together, the available experimental data suggest that a hierarchically organized stream of several visual areas is concerned with texture processing. Within this hierarchy, segmentation of texture regions is achieved by detection of salient pattern arrangements signaling region boundaries. Probable candidate areas are part of the infero-temporal pathway starting at parvocellular cell layers of the lateral geniculate nuclei (LGN) and projecting via the interblob regions of $\mathrm{V} 1$ and pale stripes of $\mathrm{V} 2$ into area $\mathrm{V} 4$ and further on to infero-temporal cortex, particularly areas TEO and TE. Areas V1 up to V4 are known to be retinotopically organized (Felleman and van Essen, 1991; Ungerleider and Haxby, 1994; Sereno et al., 1995; Peterhans, 1997; Tootell et al., 1997) and orientation selectivity is a prominent feature of cells in all these areas (Zilles and Clarke, 1997).

Based on observations from experimental investigations we propose a neural model of pre-attentive segmentation of oriented textures, attempting to integrate a large database of available anatomical and physiological data into a common theoretical framework. The key properties of the model are that (i) texture segmentation is based on boundary detection and that (ii) texture border detection is mainly achieved by cells in higher model areas. The computational mechanisms were motivated by previously proposed principles of recurrent interaction for response integration and cortical prediction (Grossberg, 1980; Mumford, 1994) and reentry processes for integration and disambiguation of localized feature measurements (Sporns et al., 1991). In particular, it shares the key processing principles of the model proposed in (Neumann and Sepp, 1999), namely (i) the bi-directional information flow between cortical areas, enabling the modulation of cell responses via feedback from higher visual areas, and (ii) the intra-area normalization of cell responses by means of competitive interaction in a spatial and orientational neighborhood. Based on the key properties and processing principles described above, we propose a neural model for texture boundary detection consisting of bi-directionally linked cortical areas V1, V2 and V4. We describe computational experiments focusing on three major aspects. First, the overall model behavior was tested systematically. The use of psychophysical stimuli enabled us to directly link observed model activation patterns with results from neurophysiological studies. Second, the precise functionality of feedback projections between cortical areas is still a focus of intensive investigations. Artificial lesions of specific model connections allowed us to identify their functional role in texture segmentation. In particular, the overall effect 


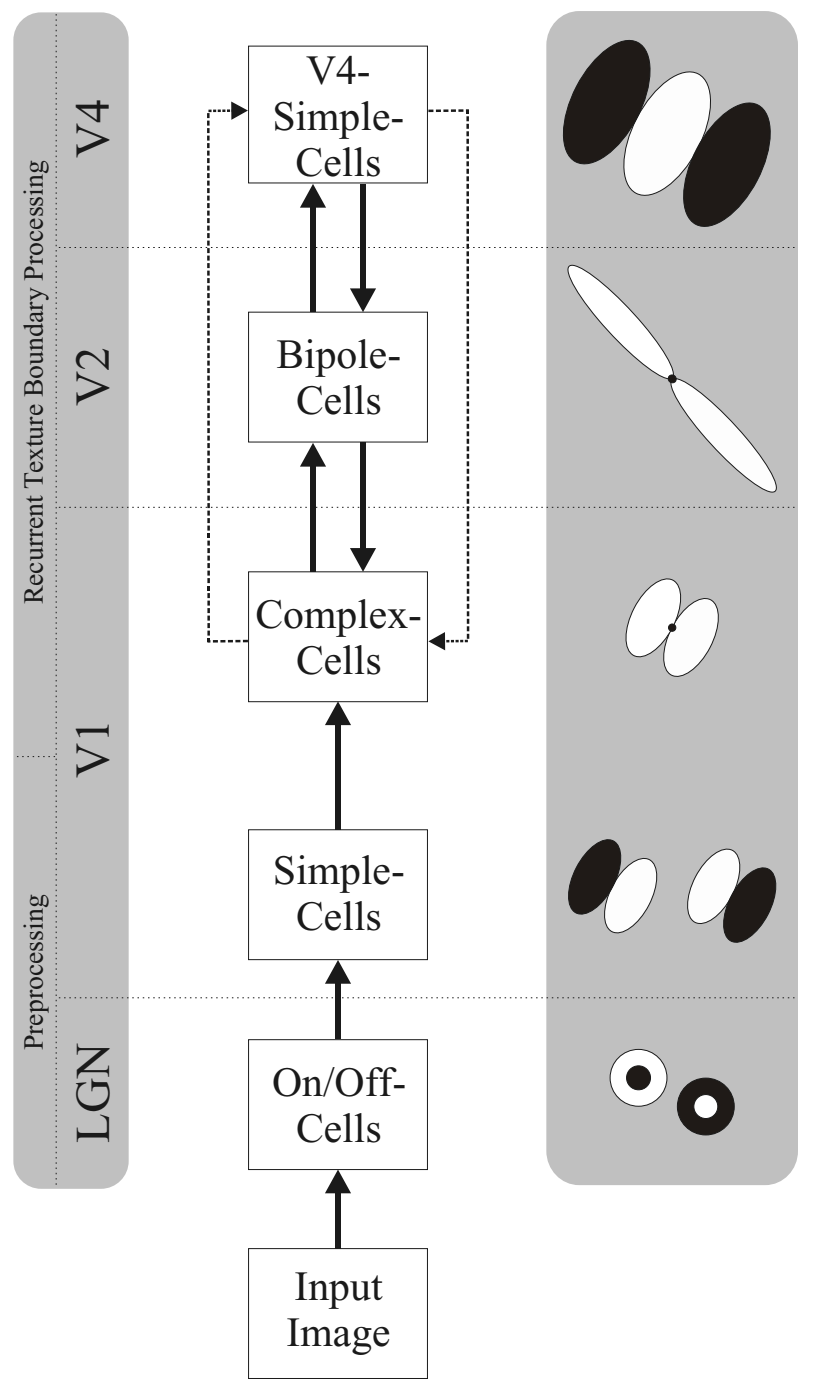

Fig. 2. Outline of the hierarchy of model areas and model cells. Receptive field kernels are sketched in the right column (white: excitatory subfields; black: inhibitory subfields). The field sizes are not drawn to scale. The dashed lines depict direct connections between model areas V1 and V4, which were used in some simulations (see Experimental Procedures sections for details).

of feedback between model areas was examined by comparing a fully connected recurrent model with a pure feedforward network. Third, weak direct bi-directional connections between V1 and V4 have been regularly reported (Felleman and van Essen, 1991; Nakamura et al., 1993; Rockland et al., 1994; Zilles and Clarke, 1997). In order to determine their functionality in the context of texture processing, we tested the impact of additional sparse direct connections between model areas V1 and V4 on the overall functionality of the neural model.

\section{EXPERIMENTAL PROCEDURES}

We propose a feed-forward and feedback model of hierarchically organized cortical areas V1, V2 and V4 which utilizes a large database of available anatomical and physiological data (Fig. 2). The model areas consist of topographical maps of mutually inter-

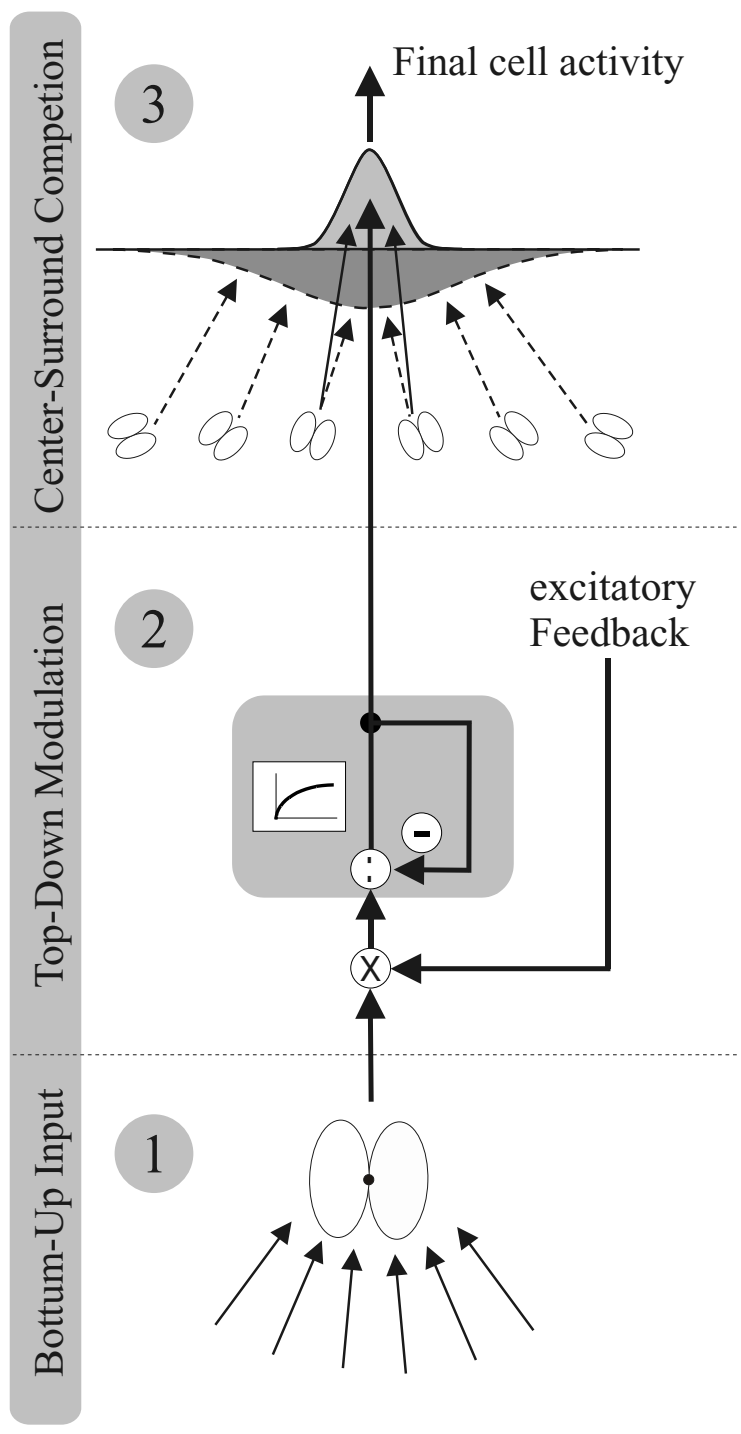

Fig. 3. Three stages of computing the activation level of a cell. (1) The bottom-up input is pooled by the cell's receptive field to generate its initial activation. (2) Multiplicative feedback interaction from higher model areas modulates the initial activation. (3) The top-down modulated activity finally undergoes a stage of shunting ON-center/OFFsurround competition in a spatial and orientational neighborhood.

connected cells with gradual activation dynamics representing the average response (firing-rate) of groups of neurons having similar selectivities. The cell's receptive fields increase as a function of hierarchically organized model areas. In accordance with data from Smith et al. (2001) their relative sizes have been parameterized in a ratio of 1:2.5:8.

\section{Model cells and dynamics}

Cells were modeled by single-compartment units of gradual activation dynamics. Such model cells represent groups, or assemblies, of individual neurons. The activation level of a cell is determined in three successive computational stages, as depicted in Fig. 3. First, the bottom-up input is pooled within the cell's receptive field to determine its initial activation. The integration is denoted by a linear equation implementing a spatial convolution. Second, the initial activation is modulated via shunting feedback 
interaction from higher model areas. Third, this top-down modulated activity undergoes a stage of shunting ON-center/OFF-surround competition between cells in a spatial and orientational neighborhood.

The dynamics of modulatory top-down interaction (second stage) is denoted by the equation

$\frac{\partial}{\partial t} l_{i \theta}^{(1)}=-\alpha_{1} l_{i \theta}^{(1)}+\left(\beta_{1}-\gamma_{1} l_{i \theta}^{(1)}\right) c_{i \theta}\left[1+C h_{i \theta}\right]$

The input activation $c_{i \theta}$ is given by the bottom-up input weighted by the receptive field kernel (first processing stage). Thus, $c_{i \theta}$ is a specific function of the cortical area the cell belongs to and is sensitive to the spatial location $i$ and to orientation $\theta$. Inspection of the equilibrated response $\left(\partial / \partial t l_{i \theta}^{(1)}=0\right)$

$l_{i \theta}^{(1)}=\frac{\beta_{1} c_{i \theta}\left[1+C h_{i \theta}\right]}{\alpha_{1}+\gamma_{1} c_{i \theta}\left[1+C h_{i \theta}\right]}$

reveals the impact of excitatory feedback activity $h_{i \theta}$ delivered by descending cortical pathways on the activation level $l_{i \theta}^{(1)}$. The strength of the excitatory feedback component is controlled by the constant $\beta_{1}$ together with the gain factor $C$. This excitatory topdown activity $h_{i \theta}$ is sensitive to spatial location and orientation. It multiplicatively enhances the initial activation $c_{i \theta}$ and is only effective at positions with non-zero initial activation. This prevents unspecific activity to spread unintentionally within the topographical map. The term $\gamma_{1} c_{i \theta}\left[1+C h_{i \theta}\right]$ is included in order to account for an upper saturation level of activation $l_{i \theta}^{(1)}$, which is given by the ratio $\beta_{1} / \gamma_{1} \cdot \alpha_{1}$ determines the rate of activity decay.

The top-down modulated activity $I^{(1)}$ of the second stage constitutes the input to the third computational stage to determine the final cell activation level $\Lambda^{(2)}$. The third stage exerts a scheme of shunting ON-center/OFF-surround competition, which is expressed by

$\frac{\partial}{\partial t} l_{i \theta}^{(2)}=-\alpha_{2} l_{i \theta}^{(2)}+\beta_{2}\left\{l^{(1)} * \psi^{+} * \Lambda^{+}\right\}_{i \theta}-\left(\delta_{2}+\zeta_{2} l_{i \theta}^{(2)}\right)\left\{l^{(1)} * \psi^{-} * \Lambda^{-}\right\}_{i \theta}$

This equation is based on a "Mexican Hat" shape for spatial as well as orientational information. The excitatory and inhibitory Gaussian weighting function, both in space and orientation, are denoted by $\Lambda^{+}, \psi^{+}, \Lambda^{-}$and $\psi^{-}$, respectively; $*$ denotes the convolution operator; $\alpha_{2}, \beta_{2}, \delta_{2}, \zeta_{2}$ are scalar constants. Examining the equilibrated response $\left(\partial / \partial t l_{i \theta}^{(2)}=0\right)$

$l_{i \theta}^{(2)}=\frac{\beta_{2}\left\{I^{(1)} * \psi^{+} * \Lambda^{+}\right\}_{i \theta}-\delta_{2}\left\{I^{(1)} * \psi^{-} * \Lambda^{-}\right\}_{i \theta}}{\alpha_{2}+\zeta_{2}\left\{I^{(1)} * \psi^{-} * \Lambda\right\}_{i \theta}}$

demonstrates the impact of the subtractive as well as divisive inhibitory surround activity. The constant $\delta_{2}$ controls the strength of subtractive inhibition given by the term $\delta_{2}\left\{l^{(1)} * \psi^{-} * \Lambda^{-}\right\}$. In addition, shunting interaction is employed to incorporate divisive inhibition by the term $\zeta_{2}\left\{I^{(1)} * \psi^{-} * \Lambda^{-}\right\}_{i \theta}$. With this scheme of competitive interaction, the initial top-down modulated activities are enhanced by contrast and normalized. The normalization achieves an activity dependent tuning of the cells' responsiveness.

In combination, the three stages of computation realize a soft-gating mechanism: the initial activities, which match the context-sensitive feedback activity from higher levels in the hierarchy, are further enhanced and inhibit cells in their neighborhood. Thus, salient texture arrangements are enhanced while at the same time spurious and perceptually irrelevant activities are suppressed. This scheme of feedback interaction followed by center-surround competition was motivated by physiological studies as well as theoretical considerations:

- The sequence of activity enhancement via feedback followed by center-surround competition has some advantages compared with a model utilizing stages in reverse order (i.e. first center-surround competition followed by feedback interaction). In a hierarchy of feed-forward connected cortical areas, it is argued that lateral inhibition is necessary to keep the activity in higher areas focused and narrow despite the larger receptive field sizes in these areas (Kastner et al., 2001). To our view, the same holds for the integration of activity from higher areas in lower areas via feedback. Consider, for example, the case that the activity distribution in a higher model area is somewhat "blurred" or broadened due to the larger receptive field sizes of the cells in that area. In a first step, feedback from this area will enhance the cell activity in a lower area at topographical positions corresponding to the same blurred region. Without center-surround competition following the feedback interaction, this in turn will lead to an activity pattern in the higher area, which tends to be even more unfocused, and so on. However, that effect is prevented by center-surround competition in the lower area narrowing the zone of activity enhancement via feedback and, in consequence, helping to spatially focus the responses to an input pattern in both the lower and the higher model area.

- The "no-strong-loops hypothesis" of Crick and Koch (1998) suggests that a directed loop between two cortical areas will not consist of two driving connections, but will use one driving and one modulatory connection in order to avoid uncontrolled oscillations of the overall system and to limit the amount of inhibition necessary to achieve a stable network behavior. Using multiplicative instead of, e.g. additive excitatory feedback is one possible implementation of such a modulatory connection. Indeed, several physiological studies indicate that feedback from higher visual areas is not capable of driving cells in lower areas, but modulates their activity (Sandell and Schiller, 1982; Mignard and Malpeli, 1991; Salin and Bullier, 1995; Hupé et al., 1998; Przybyszewski et al., 2000). For example, feedback activation alone is not sufficient to drive V1 neurons if they are not stimulated by a visual feeding input (Sandell and Schiller, 1982). Likewise, projections from the striate cortex to the LGN multiplicatively enhance responses of parvocellular neurons to grating stimuli (Przybyszewski et al., 2000). Hupé et al. (1998) demonstrated that feedback from area V5 to areas V1, V2 and V3 amplifies and facilitates neural responses in these areas.

- In the study of Hupé et al. (1998) feedback from V5 had the highest impact on cell firing rates in V3 for low saliency stimuli, but had a markedly weaker effect for high saliency stimuli. Our model dynamics mimics this behavior in that the output of a model cell is driven in saturation for strong bottom-up input and, in consequence, is hardly modulated by feedback activity in that case. However, feedback activity can substantially amplify the activation strength of a model cell in case of weak bottom-up input.

Taken together, feedback from higher model areas realizes a context-selective gain enhancement or soft-gating mechanism in our model dynamics, which facilitates initial bottom-up activity matching the "expectation" of the cells in higher model areas.

Prior to the actual simulations, the constants were empirically determined in such a way that the whole network could reach a stable activation pattern quickly after onset of input pattern presentation. In order to speed up processing, the differential equations were solved at equilibrium in response to a constant input. Initially, the activities of all model layers were set to zero. The input image was clamped and the activities of the model areas were sequentially updated. The final activation patterns were achieved after four to five iterative cycles. Each simulation was continued until iteration nine in order to visually demonstrate the stability of the solution. A comparison with results obtained by numerical integration of the model equations revealed that the use of equilibrium responses did not affect the results of the final activation patterns. The values for the constants and the standard deviations of the Gaussian kernels used in the simulations are given in Appendix A and were kept constant through all numerical experiments. 


\section{Model layers and receptive field properties}

The model consists of one subcortical area resembling LGN and three models of cortical areas V1, V2 and V4 (Fig. 2). In the following, the receptive field properties of the model cells incorporated in these areas are outlined. Please refer to Appendix B for a complete description of the mathematical equations used.

- The first two processing stages emulate roughly the functionality seen in the parvocellular layer of LGN and simple cells in V1. They were incorporated in the model for preprocessing the initial luminance distribution and their activity is determined by pure feed-forward processing. Model LGN ON and OFF cells with concentric center-surround receptive fields (Hubel and Wiesel, 1962) initially detect local luminance transitions in the input image based on a subtractive and half-wave rectified interaction between Gaussian weighted input intensities. Model V1 simple cells have elongated ON and OFF subfields to pool the input delivered by appropriately aligned LGN cells. They respond to local luminance transitions along a given orientation preference and are selective to contrast polarity (dark-light and light-dark in eight discrete orientations).

- Model V1 complex cells form the lowest level of recurrent processing for texture boundary computation. Model complex cells pool the activity of two simple cells of opposite polarity at each position. In combination, the computation performed by model LGN, simple and complex cells result in complex cell activity which is sensitive to orientation but insensitive to the direction of contrast. The output of the model V1 complex cells thus resembles that of real cortical complex cells. This output activation is subsequently modulated by top-down interaction and centersurround competition utilizing the three sequential computational stages described above.

- Model V2 bipole cells use two prolated subfields aligned along the axis of the cell's orientation preference to pool the input delivered by appropriately aligned V1 complex cells (Grossberg and Mingolla, 1985; Ross et al., 2000; Neumann and Mingolla, 2001). Model V2 bipole cells respond to luminance contrasts as well as to illusory contours, thus resembling the functional properties of contour neurons in V2 (von der Heydt et al., 1984, 1993; Heitger et al., 1998). The bipole cells combine the activities of the subfields using a soft-AND-gate, thus only generating significant responses when both fields are excited simultaneously (Neumann et al., 1999; Neumann and Sepp, 1999). The cells are capable of completing fragmented contours and respond to illusory contours induced by two or more line ends exciting both subfields. The initial responses are again modulated via top-down feedback and center-surround competition.

- Model V4 cells detect texture borders defined by activity transitions in the fields of oriented input responses generated by oriented model V1 and V2 cells. Discontinuities in the activity distribution can occur in each input orientation field (depending on the orientation of the line elements) and can themselves have different orientations (depending on the orientation of the texture boundaries). Accordingly, model V4 cells of different orientation selectivities were defined each of which having one central excitatory and two lateral inhibitory subfields all selectively integrating from the same input orientation field ( $8 \mathrm{~V} 4$ cell orientations per input orientation result in a matrix of $8 \times 8=64 \mathrm{~V} 4$ cells). All cells selective to the same input orientation undergo center-surround competition. The activities are subsequently pooled at each position for each separate input orientation and finally fed back to corresponding orientation channels in the preceding model areas.

\section{Inter-areal connections}

The information flow between visual areas V1, V2 and V4 is mainly mediated by dense bi-directional projections from $\mathrm{V} 1$ to $\mathrm{V} 2$ and from V2 to V4 (Felleman and van Essen, 1991; Zilles and Clarke, 1997). The connections between the model areas were chosen in accordance to these anatomical findings in the majority of simulations (Fig. 2). The precise functionality of feedback projections between cortical areas is still a focus of intensive investigations. Thus, a (pure feed-forward) network with artificially lesioned feedback connections was utilized in one set of simulations. Comparison of its activation patterns with those of the fully connected recurrent model allowed us to identify the role of feedback in the context of texture processing. Additionally, the selective lesion of the projections from V4 to V2 allowed to define the functional roles of areas V2 and V4 more precisely. Direct weak bi-directional projections between the areas $\mathrm{V} 1$ and $\mathrm{V} 4$ were often reported (Felleman and van Essen, 1991; Nakamura et al., 1993; Rockland et al., 1994; Zilles and Clarke, 1997). We tested the impact of sparse direct connections between model areas $\mathrm{V} 1$ and V4 on the overall functionality of the neural model. The strength of these direct top-down and bottom-up input connections was chosen to be symmetric and to be $10 \%$ or $25 \%$ of the main $\mathrm{V} 1-\mathrm{V} 2$ / $\mathrm{V} 2-\mathrm{V} 4$ connections.

\section{Stimuli}

The model behavior was systematically investigated using stimuli introduced by Nothdurft $(1985,1991)$ (e.g. Fig. 4). The use of these stimuli allows to directly compare the activation patterns observed in the network with results from psychophysical studies. Unless otherwise indicated, gray scale stimuli of sizes $270 \times 270$ were utilized containing texture arrays of $12 \times 12$ line elements. In the central region a square or bar pops out, caused by a certain orientation contrast $(\mathrm{OC})$ between the neighboring lines at the region border. Line positions were slightly varied to avoid alignment effects. An additional constant orientation shift between neighboring line elements leads to apparent "flow patterns" and thus introduces orientation noise (Fig. 5). The difficulty to detect the central pop-out area can be controlled by means of the $\mathrm{OC}$ at the border and the background orientation shift, allowing a systematic parametric examination of the activation pattern of the model network. Natural textured images were finally used to demonstrate the ability of the model to segment those images. Most images used in the simulations were black lines on white background. In order to guarantee well-defined convolution results at image boundaries and to prevent convolution artifacts, white pixels resembling the background were padded to each side of the stimulus prior to a simulation. The number of rows and columns padded was three times the standard deviation of the widest Gaussian used. This is equivalent to presenting the stimulus on a uniform white background.

\section{Quantitative measures of cell activation levels}

To systematically investigate the impact of lesions on model activation patterns, quantitative values of mean cell activation levels in model area $\mathrm{V} 4$ were obtained for several network configurations (e.g. full recurrent, pure feed-forward, without feedback from V4 to V2). For each network configuration, two sets of simulations were performed, one without background orientation noise (see example stimulus of Fig. 8a) and one with an background orientation shift of $20^{\circ}$ (see example stimulus of Fig. 8b).

For each set of simulations, the $\mathrm{OC}$ of the input texture stimulus was systematically varied between $0^{\circ}$ and $90^{\circ}$ (OC values used were $\left.0,10,15,20,25,30,40,50,70,90^{\circ}\right)$. The simulations for a specific OC were repeated for several background line orientations (background line orientations were 0 , $\left.15,30,45,60,75,90^{\circ}\right)$. This allowed us to average the activation levels over several background orientations for each value of $\mathrm{OC}$.

After each simulation we determined the mean cell activities in the $\mathrm{V} 4$ region corresponding to the inner pop-out bar (red region of model area V4 depicted in Fig. 8b) as well as the region corresponding to the surround (blue region of model area V4 

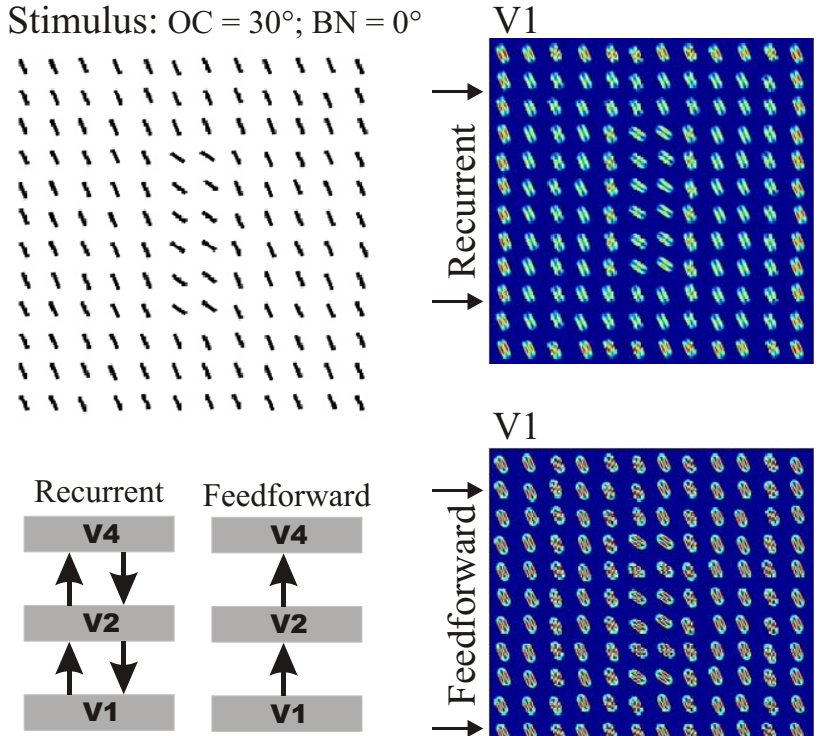
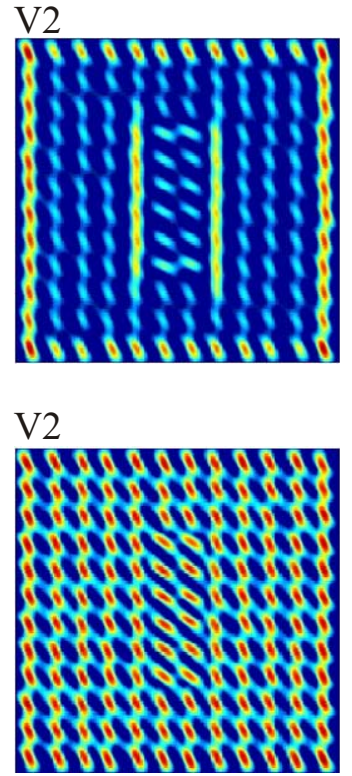

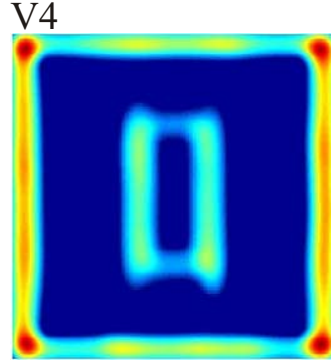

\section{V4}

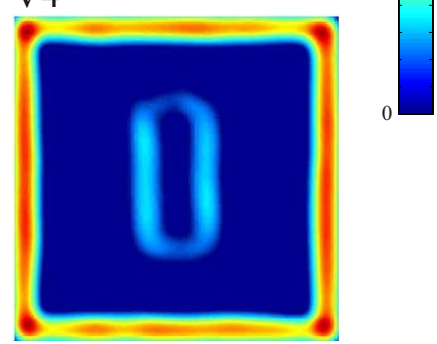

Fig. 4. Equilibrated responses summed over all orientations to a central pop-out bar. Two versions of the network are considered, namely recurrent and pure feed-forward. The feedback modulation of the recurrent model leads to a pronounced activity in model area $\mathrm{V} 4$ at positions corresponding to the border of the bar. The accentuation is evoked by the alignment of the background lines at the border of the bar in combination with the non-alignment of the central pop-out lines. This can be tested by lesioning the feedback from V2 to V1 in order to inhibit the recurrent modulation of V1 responses.

depicted in Fig. 8b). For the simulations with background orientation noise, the ratios between these two activity levels were averaged over all background line orientations for each value of OC. Finally, the mean ratios were plotted against OC. For the simula- tions without background orientation noise, only the V4 cell activation in the region corresponding to the inner pop-out bar was taken, averaged over the background orientations and the mean activity was finally plotted against OC.
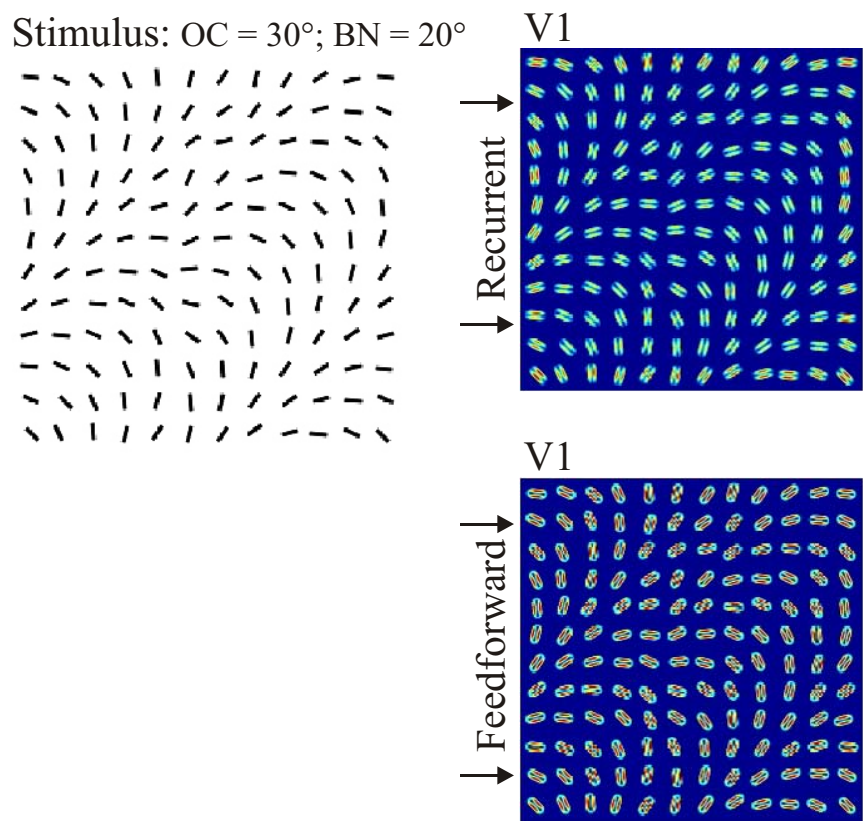
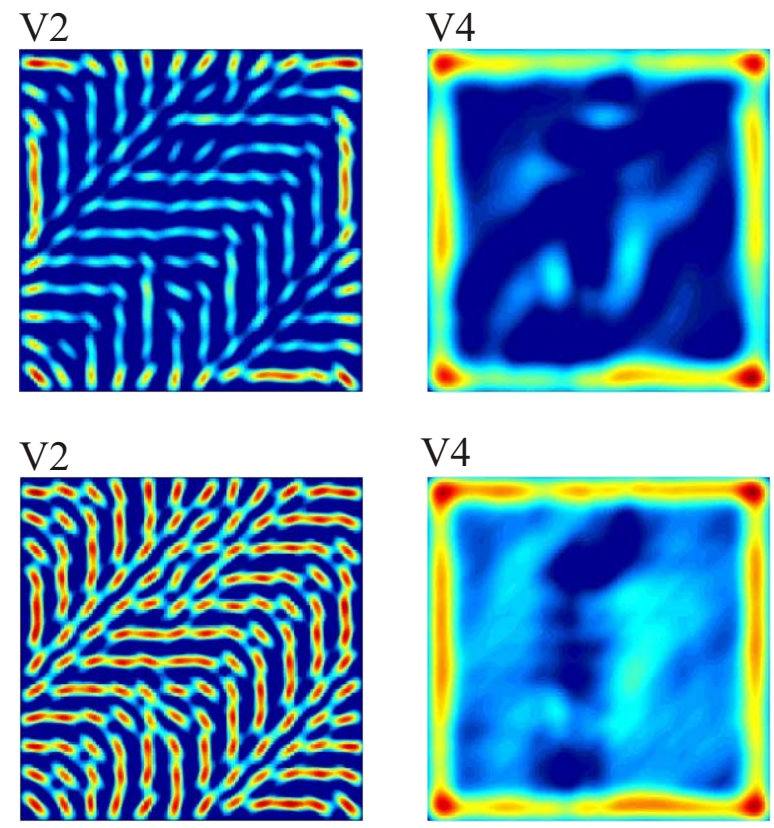

Fig. 5. Equilibrated responses of the recurrent and feed-forward nets to a central pop-out bar camouflaged by background orientation noise. Both nets fail to identify the pop-out bar, which is consistent with the subjective impression of the image. In the recurrent model, activities in model area V4 that correspond to the background flow pattern are widely suppressed. 

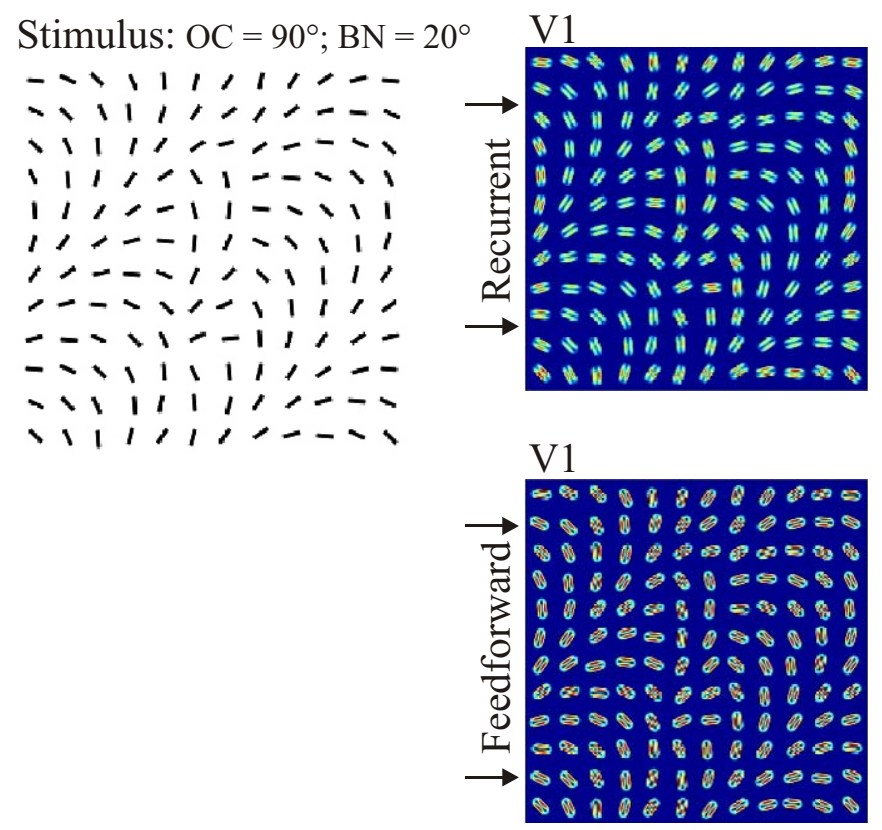
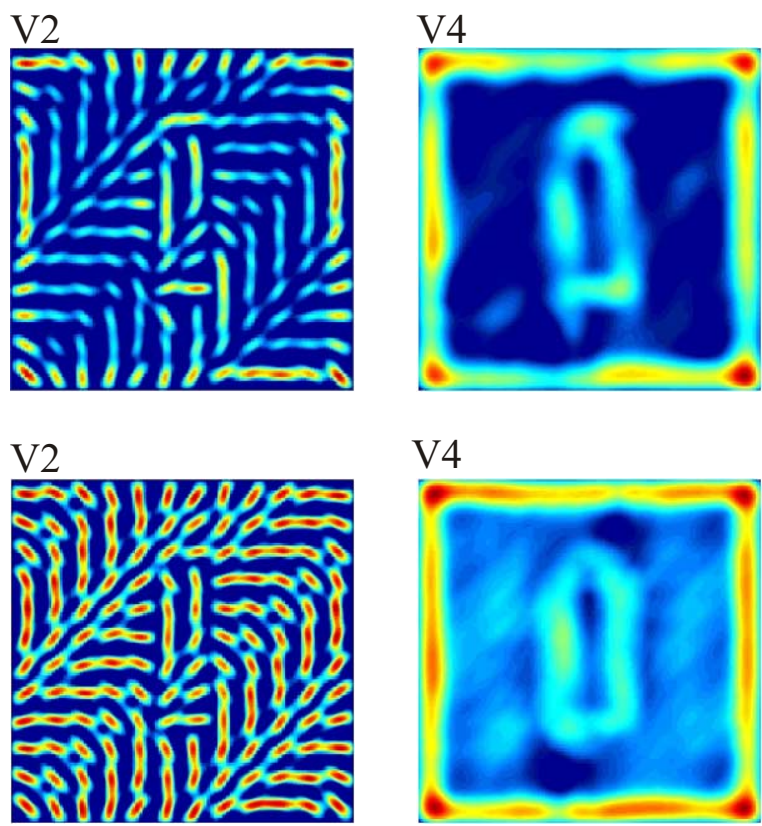

Fig. 6. Equilibrated responses of the recurrent and feed-forward versions of the network to a pop-out bar defined by a high OC in a background containing orientation noise. Feedback modulation in the recurrent model suppresses spurious activities in model area V4 introduced by the background noise and sharpens the representations of texture boundaries.

\section{RESULTS}

\section{Responses of the full recurrent model to artificial line texture stimuli}

The general computational capabilities of the full recurrent model in texture boundary detection are demonstrated in the top rows of Figs. 4-7. Shown are the equilibrated activity patterns of all model areas, for purposes of visualization summed up over all orientations at each location and subsequently scaled with respect to the maximum activity in the particular model area.

The stimulus shown in Fig. 4 consists of a vertical bar composed of oriented line items whose outline is defined by a border $\mathrm{OC}$ of $30^{\circ}$. The borders of the bar as well as the entire texture field are clearly detected by model V4 cells. The responses of model V2 cells at the borders are enhanced via modulatory feedback interaction. This enhancement in turn leads to an indirect and weaker modulation of model V1 cells, as qualitatively demonstrated by the color-coded pictures. Quantitative results of this effect will be discussed in the section concerning the sparse direct connections between $\mathrm{V} 1$ and V4.

Systematic variation of border $\mathrm{OC}$ between $0^{\circ}$ and $90^{\circ}$ reveals a sigmoidal dependency of the mean V4 activity in the region corresponding to the pop-out bar on OC (blue solid line in Fig. 8a; see Experimental Procedures section for details). The steepest increase in V4 activity signaling the outline of the pop-out bar can be observed for OCvalues between $15^{\circ}$ and $30^{\circ}$.

The introduction of a background orientation shift of $20^{\circ}$ leads to the apparent "flow pattern" visible in the stimulus of Fig. 5. The bar is still defined by a border $\mathrm{OC}$ of $30^{\circ}$, which is not sufficient to produce a significant response in model area V4. The outer border of the texture field is still clearly recognized. Some spurious cell activities due to the background orientation shift remain in the center of the texture field. Model V4 activity again reinforces the responses of the V2 and V1 cells at the border of the texture field. The visual appearance of a flow pattern corresponds with the activity distribution in model area $\mathrm{V} 2$, evoked by bipole cells grouping the bottom-up input from area $\mathrm{V} 1$ to form continuous smooth curves.

The detection of the central bar can be recovered by enhancing the border OC as shown in Fig. 6. Here, the border of the bar is clearly visible in the activity pattern of model area V4. This activity in turn modulates the responses of V2 and V1 cells at corresponding spatial positions.

The background orientation shift of $20^{\circ}$ increases the values of $\mathrm{OC}$ which are necessary for model area V4 to unambiguously represent the pop-out bar, as can be seen in Fig. 8b (blue solid line). For example, the ratio between the inner V4 activity signaling the outline of the pop-out bar and the surrounding V4 activity due to orientation noise is only marginally increased over one for an OC of $30^{\circ}$. In contrast, for the same $\mathrm{OC}$ of $30^{\circ}$ and no orientation noise the mean inner $\mathrm{V} 4$ activity unambiguously signals the popout bar (Fig. 8a), as no V4 activity due to orientation noise is present in the surrounding.

\section{Functional role of feedback projections}

In order to investigate the functional role of the top-down modulation via feedback connections we compared the recurrent model with a pure feed-forward network. The activation patterns of the feed-forward model are shown in the second rows of Figs. 4-6. As expected, the responses 
of model V1 and V2 cells to line segments are homogenous and not enhanced at the borders of the bar or the texture field. Additionally, V4 cell responses to the central bar of the stimulus of Fig. 4 is less pronounced.

In order to compare the results and influences of feedback on a quantitative basis, we display V4 activity for the central pop-out bar against border OC (cyan solid line in Fig. 8a). In the case of pure feedforward processing the activity (as a function of $\mathrm{OC}$ ) is always reduced in comparison to the full recurrent model (blue solid line) for OCvalues up to $70^{\circ}$.

Feedback helps to selectively enhance salient texture boundaries, while, at the same time, suppressing ambiguous and spurious responses. Consider, for example, the pattern in Fig. 5. A vertical bar with a OC of $30^{\circ}$ is invisible against a background orientation noise of $20^{\circ}$. While the feedforward model generates unspecific regional activity due to orientation noise the recurrent model significantly reduces such pattern activation. For a sufficient border OC of $90^{\circ}$ the central bar becomes visible (Fig. 6). The feedforward model generates coarse-grained boundary responses together with high background activation. The recurrent feedback model further sharpens boundary responses while at the same time suppresses background noise.

We calculated the ratio of mean V4 activity for the central bar against the mean background activation and display this ratio as a function of OC (Fig. 8b). The key observations are two-fold: First, the ratio computed for the feedforward model (cyan solid line) is markedly reduced in comparison to the one computed for the full recurrent model (blue solid line). Second, while the ratio of one is exceeded in the recurrent model for a border $\mathrm{OC}$ of $25^{\circ}$, a border OC of $50^{\circ}$ is necessary for the feedforward model. The result of the recurrent model corresponds to the visibility threshold measured psychophysically in (Nothdurft, 1991). In that paper, it was demonstrated that the visibility threshold (defined as $75 \%$ correct responses) for texture bars embedded in $20^{\circ}$ orientation noise ranged between $35^{\circ}$ and $40^{\circ}$ border OC. We can assume here that the texture border is detected, when the ratio between V4 center and surround activity exceeds a given threshold value. This threshold should be higher than one in order to gain noise robustness. In other words, the ratio of 1 should be reached in the model for OC values smaller than $35^{\circ}$, which is the case for the full recurrent but clearly not for the feedforward model.

It should be noted that the initial activity patterns of the recurrent model are comparable to those of the feedforward network. Consequently, the fast feed-forward processing suffices to segment simple and unambiguous image structures. The segregation of more complex scenes, however, crucially depends on recurrent processing mediated by the feedback connections and in turn takes more time to develop.

\section{Functional role of model area V2}

In contrast to model areas $\mathrm{V} 1$ and $\mathrm{V} 4$, the functional role of model area $\mathrm{V} 2$ in texture boundary processing is less obvious. Model V1 cells map the input image into a distributed representation in several (in our case eight) orientation fields. Model V4 cells detect discontinuities in these orientation fields and establish region boundaries along different orientations. Therefore, both areas are mandatory for the segregation of oriented textures. In the model, V2 bipole cells selectively enhance those V1 complex cell responses which form smooth flow lines from several adjacent line elements (see Figs. 4-6). The influence of this alignment effect on the capabilities of the complete model in texture boundary processing can be best tested by comparing a model version with artificially lesioned V4-V2 feedback connections against the full recurrent model. After lesioning, model area V4 looses its ability to modulate the cell activity in lower areas, which in turn enables unhindered alignment of line elements by $\mathrm{V} 2$ bipole cells. The resulting activity patterns are exemplarily demonstrated in Fig. 7 using a stimulus which consists of lines which are tilted $+15^{\circ}$ in the background area and $-15^{\circ}$ in the pop-out area with respect to the vertical. Model V2 cells tend to align the line elements of the background and the pop out region to vertical continuous activation patterns and thus reduce the initial OC. Model V4 cells attempt to enhance the initial contrast via feedback. After lesioning the model V4-V2 feedback connections, V4 looses its ability to preserve the contrast and the boundary activations in V4 are no longer maintained (second row of Fig. 7). This attenuation effect can also be observed when systematically investigating the model V4 activity in dependence of the border OC (green dashed lines in Fig. 8a and $b$ ). In the case of no background orientation noise (Fig. 8a), active V2-V1 feedback reduces the V4 activity for the pop-out bar below the activation strength of the feed-forward model. This indicates that the alignment mediated by model area V2 diminishes the capabilities of the overall model in texture boundary detection.

\section{Effects of direct projections between areas V1 and V4 on V4 activation strength}

The effect of direct bi-directional connections between model areas $\mathrm{V} 4$ and $\mathrm{V} 1$ in addition to the pair-wise V1-V2 and V2-V4 connectivities, respectively, has been examined using a model in which the direct bidirectional input constituted $25 \%$ of the total input to areas V1 and V4. As can be seen from Fig. 8a, the direct connections lead to an increased steepness of the sigmoidal curve of inner V4 activation on the $\mathrm{OC}$ in the case of no orientation noise (purple dashed line). Furthermore, direct bi-directional projections increase the inner region to surround ratio in the case of $20^{\circ}$ background orientation noise (Fig. $8 \mathrm{~b}$ ). In the previous section it was demonstrated that model area V2 diminishes model V4 cell activities at texture borders. This corresponds well with increased V4 activations in the case of direct V1-V4 projections bypassing model area $\mathrm{V} 2$ and thus reducing its influence on model area V4. 

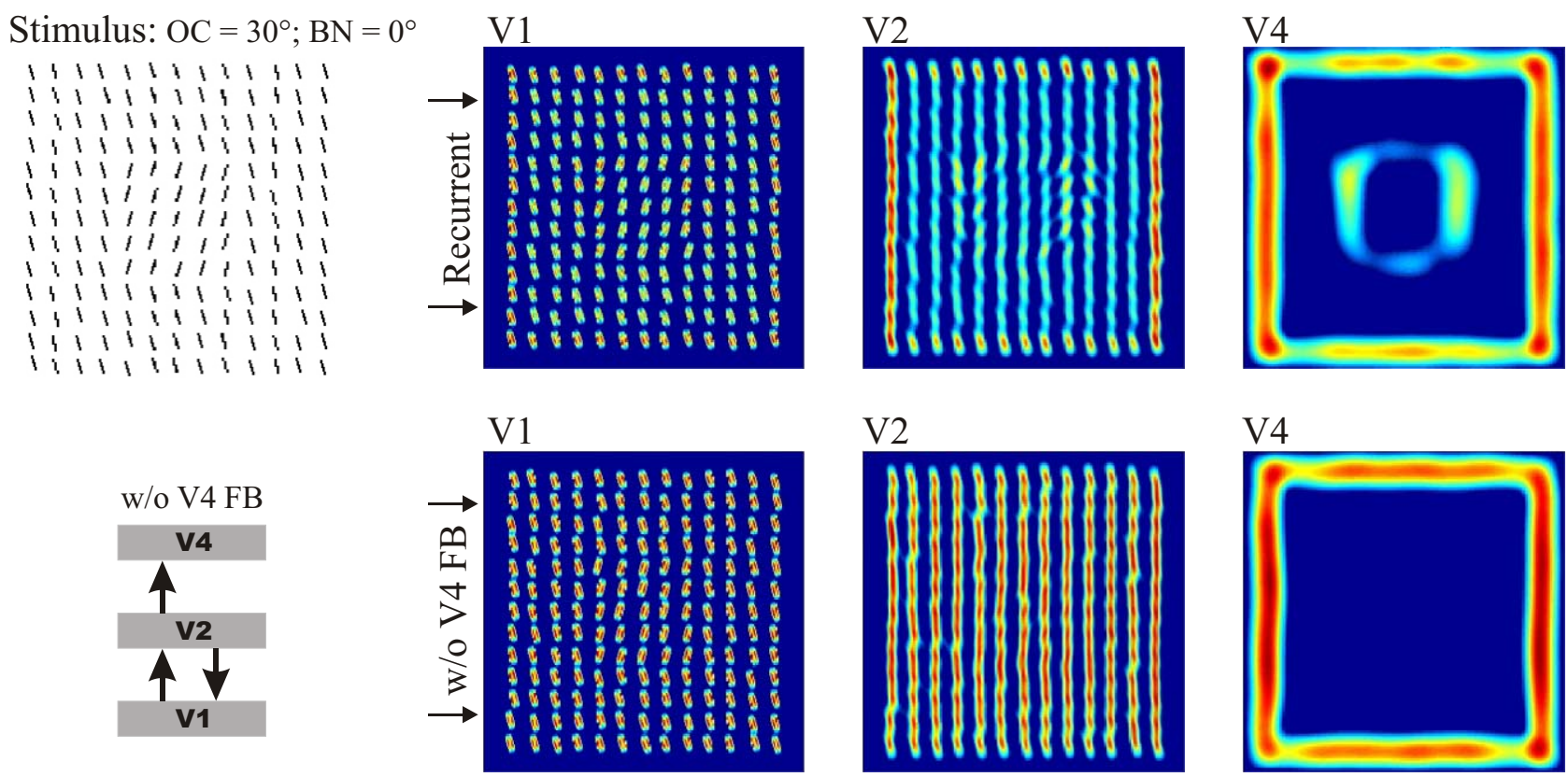

Fig. 7. Equilibrated responses of the full recurrent network and a network without feedback from model area V4 to a central pop-out square (stimulus size $300 \times 300$ pixel). The responses in model area V4 to the horizontal borders of the square are attenuated due to alignment effects in model area V2. Without feedback from model V4, alignment leads to a complete suppression of the square region.

\section{Effects of direct projections between areas V1 and V4 on V1 complex cell responses}

The effect of direct bi-directional V1-V4 connections on V1 complex cell responses has been examined using the stimulus shown in Fig. 9. A central pop-out square is defined by a border OC of $90^{\circ}$. Since gray scale pictures can only provide a coarse impression of the absolute height of cell response levels, quantitative values of V1 cell activities were displayed as follows: We calculated the mean V1 activation levels in each column of spatial locations within the region indicated by the dashed lines. This leads to activation patterns along the abscissa as shown in the bottom pictures of Fig. 9.

In this experiment we systematically varied the relative strengths of the bi-directional connections between model areas V1 and V4. In particular, three different conditions were tested, namely $0 \%, 10 \%$ and $25 \%$ (numbers in percent of total modulatory input strength to $\mathrm{V} 1$ and $\mathrm{V} 4$, respectively). The difference between the activities of the background line elements at the border of the square as well as the adjacent line column was determined (Fig. 9). When considering the model without direct $\mathrm{V} 1-\mathrm{V} 4$ connections, the indirect interactions via model $\mathrm{V} 2$ enhance the model $\mathrm{V} 1$ complex cell responses by $25 \%$ at the texture square border. The direct bidirectional connections between model areas V1 and V4 further increase the responses of the complex cells at the border (additional 18\% increase at 10\% direct V1-V4 connection strength; $27 \%$ increase at $25 \%$ direct connection strength as compared with the model without direct V1-V4 connections). In other words, $10 \%$ direct V1-V4 interaction nearly doubles the border enhance- ment effect in $\mathrm{V} 1$ compared with the model without V1-V4 connections.

\section{Segmentation of real-world images: an example}

In the present study, the use of stimuli adapted from psychophysical studies enabled us to systematically investigate and characterize the model behavior. Such stimuli show rather idealized structures compared with real-world textures, making it difficult to estimate the computational capacities of the model in segmenting more complex images. However, we successfully conducted a series of simulations using real-world pictures containing regions of oriented textures. Consider, for example, the responses of the full recurrent model to the Dalmatian (Fig. 1) in Fig. 10. The dog cannot be segregated from ground by detecting a continuous luminance transition to delineate its outline, but has to be identified by means of the changing texture. Consequently, model $\mathrm{V} 1$ cells sensitive to luminance transitions that lie at the border between the dog and the background show cluttered and fragmented activities. Model areas V2 and V4 succeed in recovering the shape of the dog in large parts by filling in the gaps of activity patterns from V1 and detect the change between the regular texture in the background and the random black dots of the dog's skin. As can be seen when comparing the results of the full recurrent and the feed-forward nets, model V4 cells detect the border of the dog as well as the border of the entire image. Model V4 cells in turn enhance the responses of model V2 cells at these positions via modulatory feedback interaction. 


\section{No Background Orientation Noise}

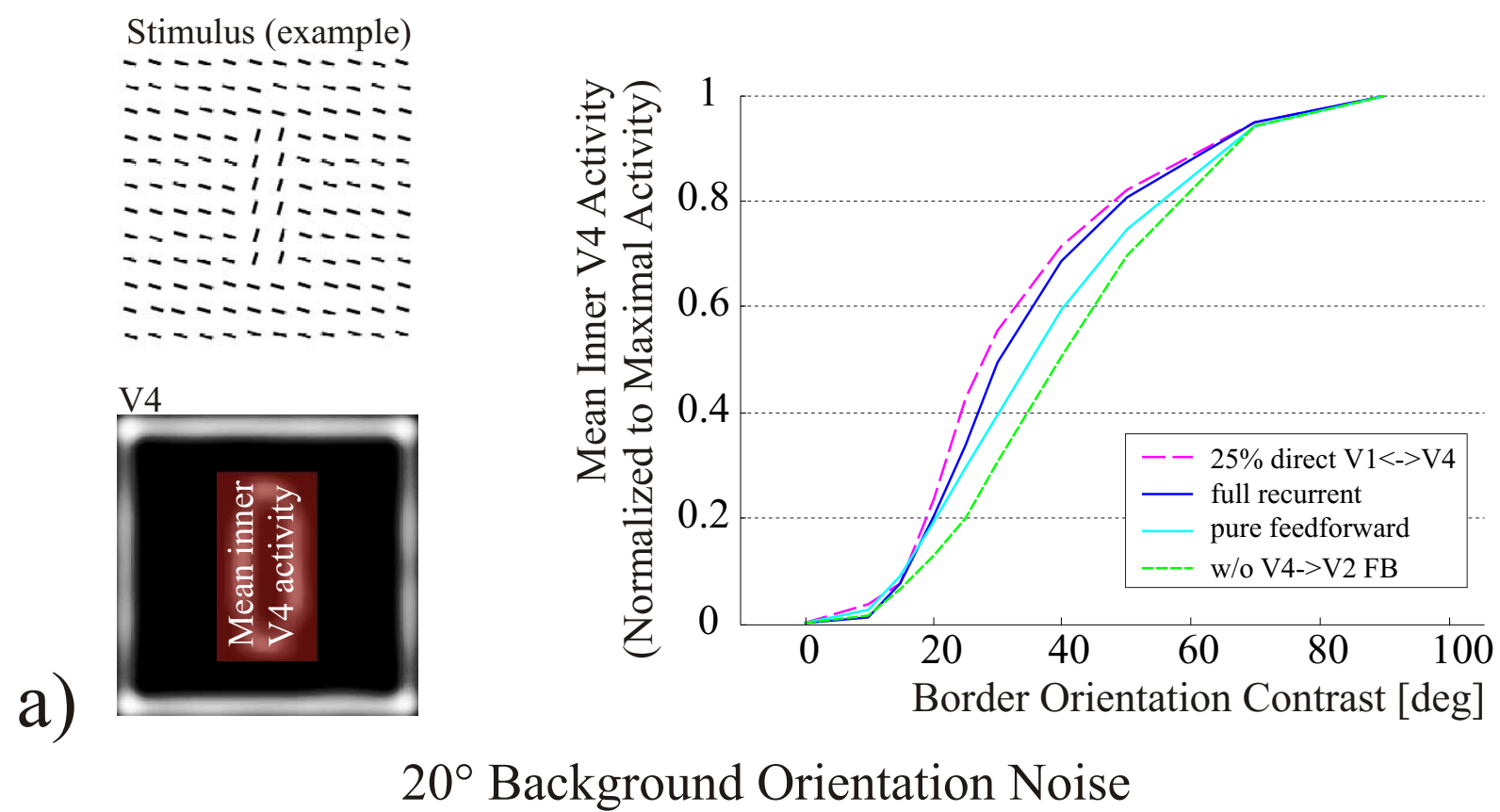

Stimulus (example)
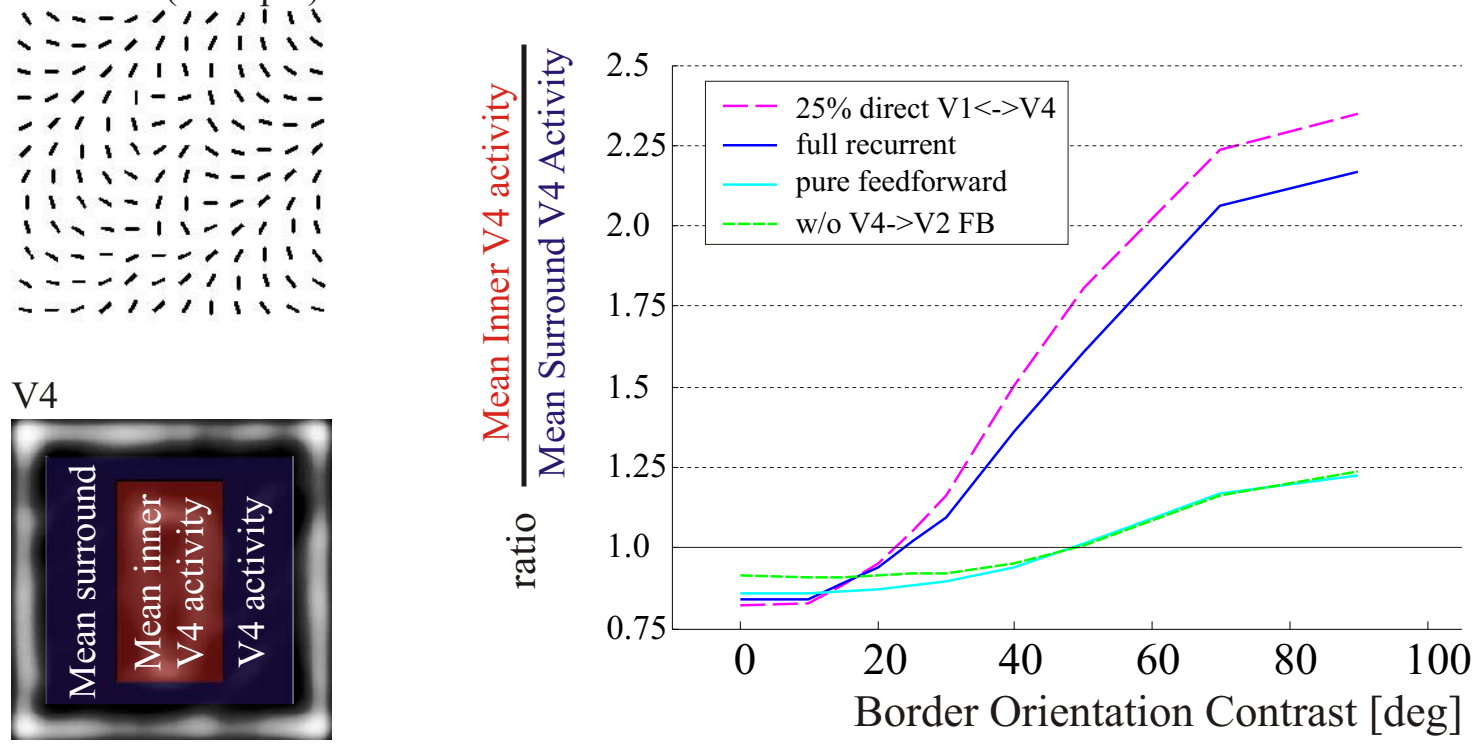

Fig. 8. Systematic investigation of model V4 activation patterns in dependence of the border OC. Four network configurations are considered, namely the full recurrent model, a model with lesioned feedback connections (pure feed-forward), a model with selective lesions of the V4-V2 feedback connections (w/o V4->V2 FB) and a model in which direct bi-directional V1-V4 input constitutes $25 \%$ of the overall input to that areas (25\% direct $\mathrm{V} 1<->\mathrm{V} 4)$. a) No background orientation noise. The mean inner V4 activity in dependence of OC is depicted (normalized to the maximal activity at $\left.\mathrm{OC} 90^{\circ}\right)$; b) $20^{\circ}$ background orientation shift. The ratio of mean V4 activity for the central bar against mean background activation is depicted.

\section{DISCUSSION}

\section{Summary of findings}

Since the early works of Beck (1966) and Julesz (1965), respectively, a variety of studies has been conducted in order to elucidate the mechanisms underlying texture segmentation. This paper integrates their results into a common computational framework, allowing to identify the functional roles of visual areas and inter-connections that are most likely involved. According to the known 

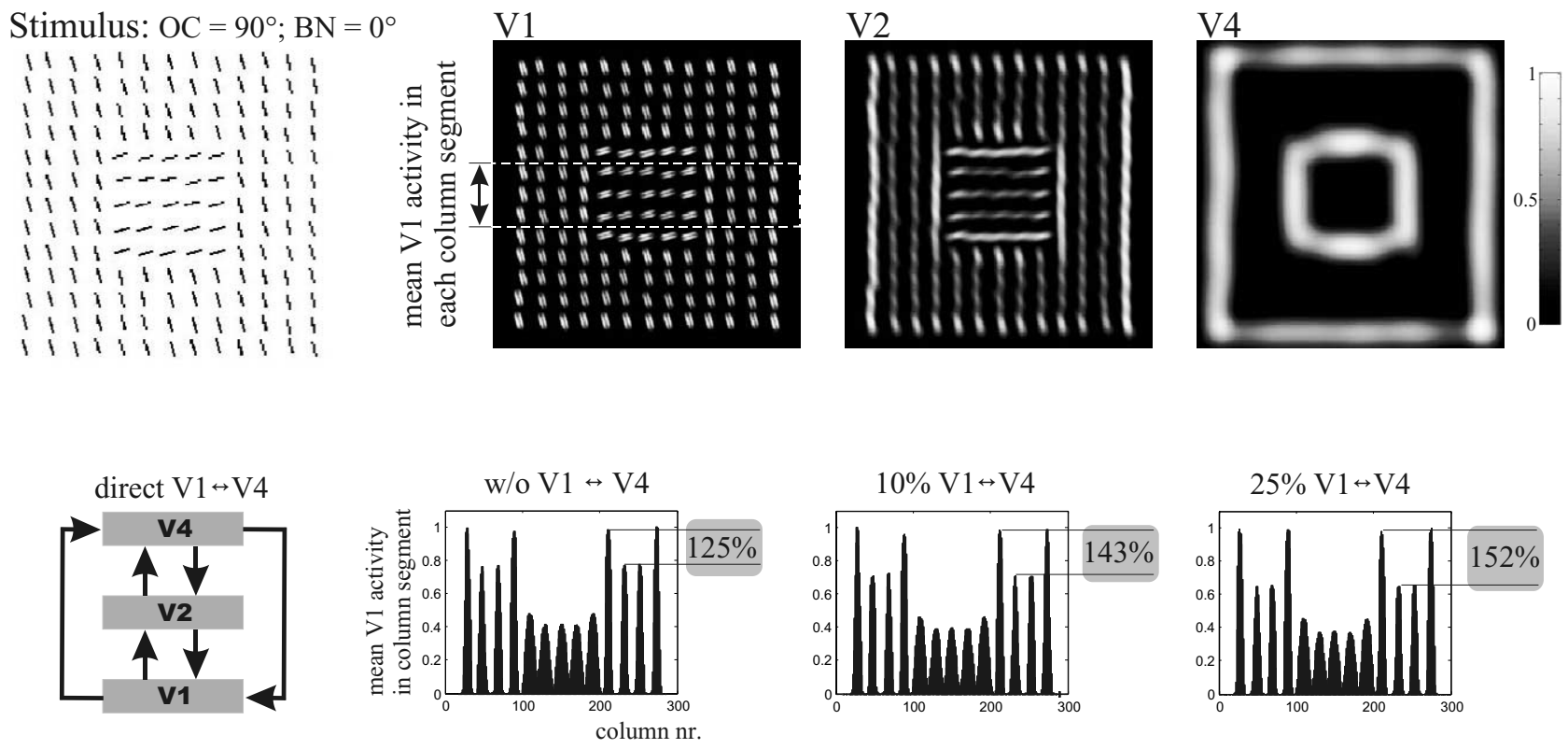

Fig. 9. Effects of additional direct connections from V4 to V1 (stimulus size $300 \times 300$ pixel). Model V1 complex cell responses of each column segment in the region denoted by the dotted white lines were summed up, leading to the response profiles depicted in the three lower illustrations. The activity of the background line elements at the border of the square was compared with the cell activity evoked by the next column of lines. Additional direct feedback from model V4 significantly increases the observed border enhancement effect.

anatomical data (Felleman and van Essen, 1991; Ungerleider and Haxby, 1994; Peterhans, 1997), the model consists of three hierarchically organized and bi-directionally linked cortical areas. The boundaries of oriented textures are detected via recurrent processing within the hierarchy. The properties of the model were demonstrated by the use of stimuli containing textures of oriented lines, which were adopted from psychophysical studies. Several sets of simulations have been performed in order to systematically investigate the model behavior for texture boundary detection. First, we demonstrated that the activation patterns observed in area V4 of the full recurrent model successfully signal the presence or absence of texture borders corresponding to the subjective impression of the stimuli. The comparison of the full recurrent model with a pure feed-forward network helped to clarify the role of feedback in the context of texture boundary processing, namely the suppression of spurious activities due to orientation noise and the enhancement and sharpening of salient texture borders. Furthermore, simulating selective lesions of the V4-V2 feedback connections permitted to determine the influence of model area V2 on the capabilities of the complete model in texture segmentation. Systematic investigation of the model $\mathrm{V} 4$ activation patterns demonstrated that the alignment generated by V2 bipole cells tends to attenuate the $\mathrm{V} 4$ responses to texture borders. In the last set of experiments the effect of direct weak connections between model areas V1 and V4 was demonstrated, namely an enhancement of V4 and V1 cell responses at texture borders.

\section{Relation to psychophysical data}

Experiments by Nothdurft $(1985,1991)$ investigated the detection of borders defined by high contrasts along a particular feature dimension. Using oriented textures, he showed that the detectability of a central region (a bar composed of oriented line items) depends on the $\mathrm{OC}$ at the region border. The critical range of $\mathrm{OC}$, in which detectability changes from invisibility to reliable identification, was $0^{\circ}$ to $30^{\circ}$ in the case of no background orientation noise. Likewise, our model shows a sigmoid-like dependence of inner V4 activity delineating the outline of the pop-out bar on the OC with the steepest increase for OC-values up to $30^{\circ}$ (blue solid line in Fig. 8a). Furthermore, Nothdurft (1985) demonstrated that OC-values necessary for a reliable identification of the pop-out bar increase with increasing background orientation noise. Similarly, for a background orientation shift of $20^{\circ}$, the ratio of mean V4 activity for the central bar against mean background activation is significantly increased above one only for OC-values greater than $30^{\circ}$ (blue solid line in Fig. 8b). It is important to note that high ratios between central and background V4 activity crucially depend on the ability of feedback to modulate the cell activation in lower model areas (the cyan solid line in Fig. $8 \mathrm{~b}$ depicts the behavior of the pure feed-forward model). A more detailed comparison of model V4 activation patterns with psychophysical curves using further values of background orientation shifts will be presented elsewhere.

Nothdurft (1992) demonstrated that alignment effects do exert influence on the detectability of a central pop-out bar. He concluded that "alignment of element orientation 

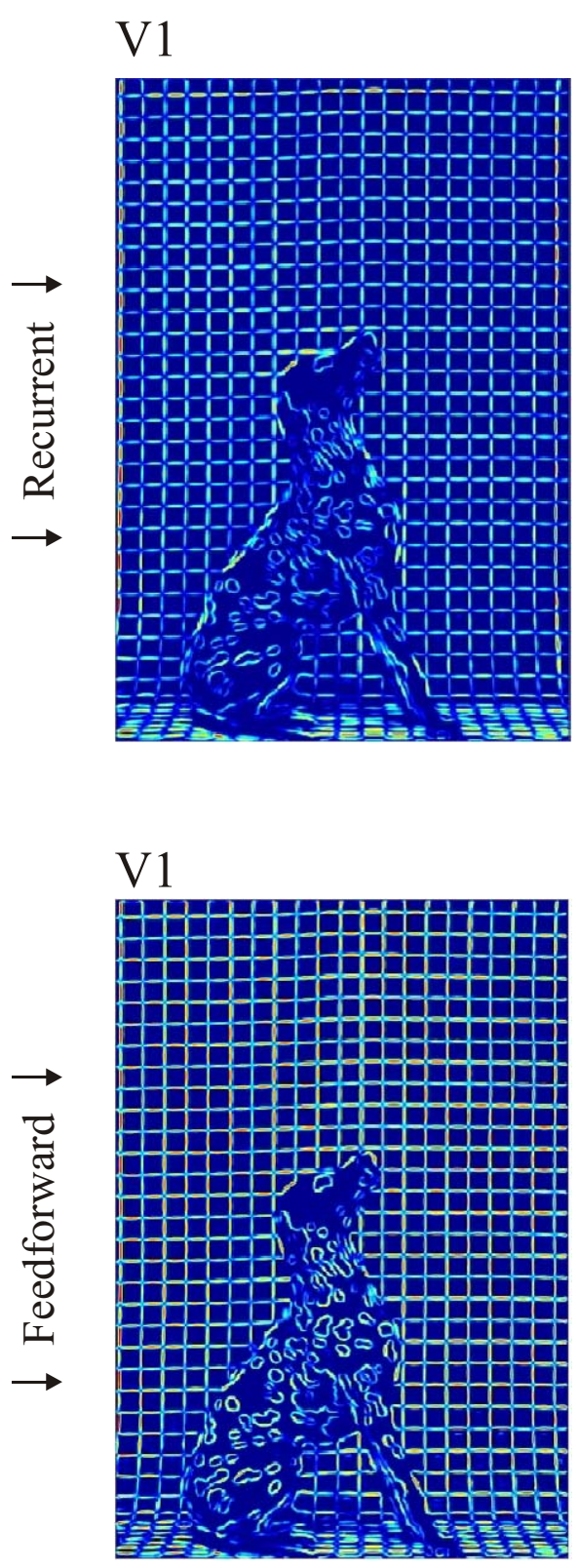

\section{V2}

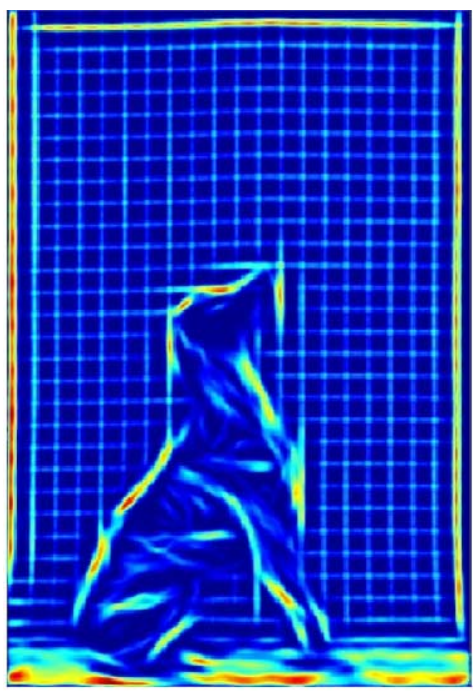

V2

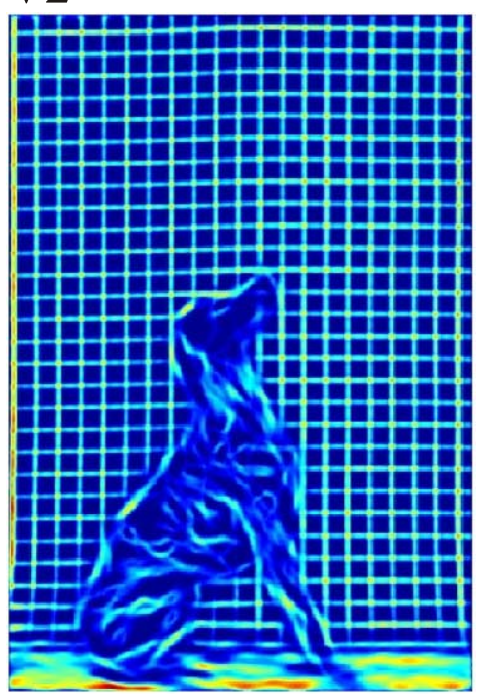

V4

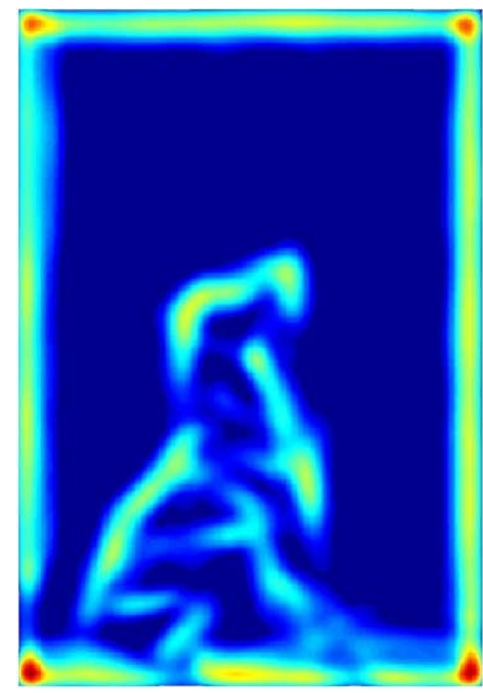

V4

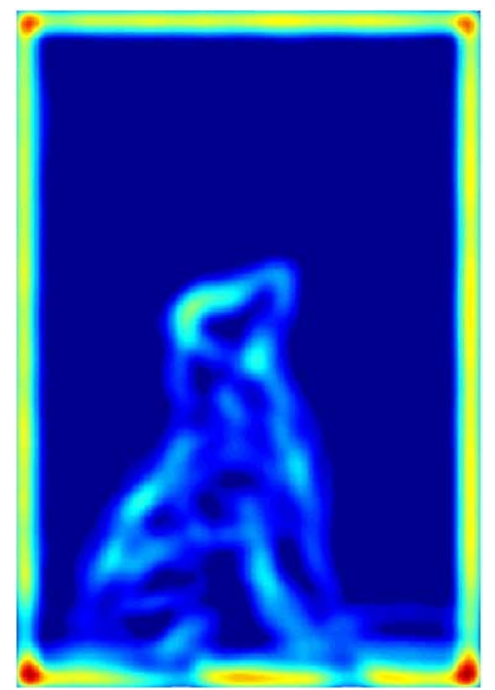

Fig. 10. Equilibrated responses of the model to the image of Fig. 1 summed over all complex cell orientations. The cells of model area V1 do not succeed in signaling a smooth continuous outline of the dog. However, model V4 cells detect the change in texture and recover major parts of the border of the dog as well as the border of the entire image. The comparison of the full recurrent with the feed-forward model demonstrates the enhancement of model V2 activity at figural borders by model V4 activity via modulatory feedback interaction.

with that of the (long) texture border helped in detection of the bar" (Nothdurft, 1992, p. 361). In our model, alignment effects lead to spatial long-range groupings by V2 bipole cells which in turn reduce the V4 activity at region borders in the case of a common alignment of the lines across these borders. This is demonstrated in Fig. 7. Alignment effects of items processed by model V2 cells lead to highly reduced V4 activities at the horizontal borders when compared with the vertical borders of a central pop-out square. Consequently, orienting the lines perpendicular to the long borders of a pop-out bar enables the V2 bipole cells to act across these long borders and leads to a markedly overall suppression of model V4 activities delineating the bar. This effect is prevented by aligning the line elements parallel to the long borders, since model V2 cells can only reduce V4 activities at short borders in this case. To conclude, the experimental findings of alignment effects influencing the detectability of a central pop-out bar can be traced back to the activity of model V2 bipole cells as the most probable neural origin.

Taken together, our simulations were able to reproduce key findings from psychophysical investigations of texture segregation. This supports the view suggested by Nothdurft $(1985,1991,1992)$ that segmentation of texture 
regions proceeds by means of boundary detection that is based on discontinuities along feature dimensions rather than grouping (or clustering) of similar feature elements. Model cells in V4 detect discontinuities in the spatial arrangement of responses in the orientation fields of model areas V1 and V2. Feedback from model area V4 enables the suppression of ambiguous responses and the enhancement of activity at discontinuities to signal salient image structures.

\section{The role of $\mathrm{V} 1$ in texture segmentation}

Several studies using single- or multi-unit recordings in the primary visual cortex of monkeys give evidence that $\mathrm{V} 1$ is involved in the processing of texture boundaries. For example, Gallant et al. (1995) reported that activity of a subsample of orientation selective neurons is significantly enhanced at the border of a bar defined by line items with a border $\mathrm{OC}$ of $90^{\circ}$. An enhancement of $\mathrm{V} 1$ cell activity at texture borders together with a suppression of cell activity by homogeneous texture surrounds was reconfirmed by the studies of Nothdurft et al. (1999, 2000). Likewise, Lamme et al. (1998, 1999) showed that multi-unit activity is strongly enhanced at the border of a square that was defined by an $\mathrm{OC}$ of $90^{\circ}$. Lamme et al. (1998) reported that the enhancement effect was still observable when substantial parts of the extrastriate cortex were ablated. This led these authors to the conclusion that cells in V1 can detect texture boundaries even without top-down input, probably based upon processes mediated by intra-areal horizontal long-range connections.

Hupé et al. (2001) reported that the response modulation in V1 is not affected by the inactivation of V2 by GABA injections, which indicates that modulation of $V 1$ mainly depends on lateral interactions within V1. However, the stimulus used there was a single pop-out line being either parallel or perpendicular to the surrounding. Suppression instead of enhancement effects were observed as the main surround effect. It might be that the chosen stimulus is not the best configuration to study feedback via longrange connections. Other studies using similar stimuli, or examining the effect of an isolated presentation of the surround on spontaneous cell activities confirm the initial inhibitory component. However, they also report an additional late facilitatory component (Li et al., 2000, 2001). In that paper, it was proposed by the authors that facilitation is mediated by feedback connections from extrastriate areas, whereas horizontal connections within V1 contribute to the initial suppression.

Taken together, the above studies indicate that the primary visual cortex appears to be involved in texture border detection. As such a task requires integration over wide areas in the visual input, the horizontal long-range connections within $\mathrm{V} 1$ are good candidates underlying the observed border enhancement effects. Computational models of V1 long-range interactions have been proposed that are able to detect texture borders in the case of high border OCs and homogeneous background texture regions (Li, 1998; Hansen and Neumann, 1999). Hansen and Neumann (1999) showed that this circuitry of long- range interaction is capable of contour enhancement and reliable corner detection, thus building a preliminary stage for perceptual grouping and figure-ground segmentation. Li's model of striate cortex also incorporates long-range connections to achieve contour enhancement. In addition, the model is able to detect the borders of simplified texture arrangements with high OCs. In most of the electrophysiological studies cited above, stimuli were employed that either consisted of a single perpendicular line element placed in an iso-oriented surround, or of texture regions with perpendicular line elements with respect to the adjacent regions. In these cases of localized high border OCs, the contour enhancement within $\mathrm{V} 1$ together with the partial loss of lateral inhibition by iso-oriented lines at the texture border can account for the observed border enhancement effect.

However, there are some factors that limit the putative role of $\mathrm{V} 1$ cells in texture border detection. First, their spatial field of activity integration is rather small even when considering horizontal long-range connections. Cavanaugh et al. $(2002 a, b)$ calculated cortical magnification factors representing the receptive field sizes of $\mathrm{V} 1$ cells in terms of cortical distances in $\mathrm{mm}$. They demonstrated that the majority of receptive field radii fell within a range of approximately $3-4 \mathrm{~mm}$ on the cortical map. Horizontal long-range connections in V1 span up to distances of 8 mm (Gilbert and Wiesel, 1989; Hirsch and Gilbert, 1991). In other words, the diameter of activity integration via horizontal long-range connections is (very roughly estimated) maximal two to three times the diameter of a cell's receptive field. However, this is still about three to four times smaller than the receptive field of a V4 cell (Smith et al., 2001). The second limiting factor stems from the spatial anisotropy of the $\mathrm{V} 1$ long-range connections. It has been demonstrated that the response of a V1 complex cell to an optimally oriented line element inside its receptive field is markedly influenced by the relative orientation and spatial position of surrounding lines (Kapadia et al., 1995, 2000). In particular, facilitation can be obtained by appropriately aligning the surrounding lines along the axis of the cell's orientation preference. In consequence, it is likely to assume that the enhancement of V1 complex cell responses at texture borders via horizontal long-range interactions is notably affected by the orientation of the line elements constituting the texture regions. However, a robust identification of texture borders crucially depends on the reliable identification of meaningful orientation discontinuities, and should be widely independent of this effect. Third, an enhanced cell firing rate in V1 is not sufficient for an unambiguous representation of region borders. Higher firing rates can be obtained by a variety of image configurations (e.g. line segments constituting a non-closed smooth contour; Kapadia et al., 1995) not necessarily representing a region border. However, an unambiguous border representation is necessary in that in can serve as starting point for figure-ground segmentation.

Taken together, we propose that horizontal long-range projections within V1 implement a powerful and multi-purpose preprocessing mechanism, e.g. to enhance the sig- 
nal-to-noise ratio, which may suffice for texture border detection in those cases where high border OCs occur isolated and localized, and where background regions show no orientation shift. The reliable formation of texture boundaries in the case of background noise requires integration utilizing a wide spatial and orientation neighborhood and is thus more likely the function of a higher cortical area. In this case, an observed border enhancement effect in V1 should be mainly due to feedback from higher areas.

In the present paper, we exclusively focus on the detection of texture borders by cells in higher visual areas and do not consider horizontal connections within V1. This restriction allowed us to investigate the contribution of higher areas along the ventral form pathway in texture processing and to facilitate the localization of the neural origin of observed modulatory effects. Furthermore, more elaborate texture patterns could be utilized this way. However, it is important to note that our model of texture boundary processing is not incompatible to models using V1 long-range connections. Instead, the model of Hansen and Neumann (1999) shares the same kind of cell dynamics and processing principles as presented in this paper and could easily be integrated in our hierarchy of model areas, just as the V2 long-range integration adopted from (Neumann and Sepp, 1999). In this case, the long-range computations in model area V1 would create some kind of "saliency map" (as proposed in Li, 2002), indicating the likely important image locations by an enhanced cell firing rate. This map would serve as pre-processed input for the higher model areas (in particular V4), allowing a faster detection of the region borders. For simple input stimuli, the cell responses in model area $\mathrm{V} 1$ to line elements at the texture borders would be increased right from the start of the simulation via (the rather fast) horizontal long-range interactions. This increase of activation would be passed on to model areas V2 and V4 via feed-forward processing at the corresponding topographical positions. Consequently, the modulation of cell activity in model areas $\mathrm{V} 1$ and V2 would not only depend on feedback from V4, but the overall model would also reach its saturated final activation pattern more quickly after a few iterations. However, the current paper serves as a clear-cut examination of the likely role of higher visual areas in texture boundary processing, which is a necessary prerequisite to study the interaction of horizontal V1 long-range processing and feedback modulation from higher model areas in further investigations.

\section{The role of higher visual areas in texture segmentation}

Results from several studies demonstrate that extrastriate areas play a crucial role in texture segregation. For example, Lamme et al. (1999) reported that multi-unit activity is moderately pronounced at positions belonging to the surface of a central pop-out square. This modulation was delayed relative to the enhancement effect at the texture border and was abolished by ablation of extrastriate visual areas. This indicates that a hierarchy of visual areas rather than the primary visual cortex alone is involved in texture segmentation and figure-ground separation.

Monkeys with lesioned area V4 were severely impaired in the perception of texture-defined contours as well as illusory contours (de Weerd et al., 1996; Merigan, 1996, 2000). In the study of Merigan (2000), the V4 lesioned monkeys were still able to detect the presence of high OCs of $90^{\circ}$ in the stimuli. However, they were no longer able to identify the orientation of a pop-out bar composed of several line elements, i.e. they could not recover the shape of the bar outlined by the texture border.

The involvement of higher cortical areas in texture segregation is also demonstrated by a recent functional brain-imaging study of Kastner et al. (2000). In contrast to lower visual areas, activities in areas V4 and TEO were significantly increased during presentation of texture stimuli containing several region borders compared with homogeneous textures. It is important to note that functional brain-imaging studies that investigate the perception of illusory or motion-defined contours showed the same tendency of increased activation strength in higher visual areas as compared with lower areas (Reppas et al., 1997; Mendola et al., 1999).

Taken together, the above cited studies strongly suggest that several visual areas-rather than V1 in isolation-are engaged in texture segmentation. However, these studies do not allow to identify the functional roles of the involved areas. For example, the mechanism of texture segmentation can be further subdivided with respect to two functional aspects, namely the detection of texture borders followed by the representation of texture surfaces. It is difficult to conclude from the above-cited studies to which proportion a specific higher visual area (and in particular V4) contributes to one of these two subtasks. Furthermore, it remains unclear to what extent the lateral interactions within area $\mathrm{V} 1$ or the feedback projections from higher cortical areas contribute to the observed border enhancement effect in V1. Based on the results of our simulation study, we propose that within the hierarchy of ventral visual areas, V4 integrates the pre-processed information from areas $\mathrm{V} 1$ and $\mathrm{V} 2$ to build a coherent neural representation of surface boundaries. Based on this border representation, V4 and probably even higher areas, such as TEO, can build up a robust representation of surfaces and the background. Unlike V1 in isolation, the full hierarchy achieves robust border detection even in noisy situations, e.g. when the texture stimulus contains orientation noise or when the spatial density of the texture line elements is low. It is worth noting that a similar effect has already been demonstrated by Hupé et al. (1998) in the dorsal pathway of motion processing, showing that feedback from MT had the greatest impact on cell activity in V3 when using low salience stimuli.

The view that at least a portion of V4 cells is selective to borders is supported by a recent electrophysiological study (Pasupathy and Connor, 2001). It was demonstrated that a significant amount of $\mathrm{V} 4$ cells selectively responds to boundaries of complex shapes defined by luminance transitions at specific positions in the stimuli. In a recent study, 
Pollen et al. (2002) investigated the interaction of bar and grating stimuli in single cell V4 responses. The key observations were that (1) individual neurons are predominantly selective to single input orientations and that (2) RF obey a spatial center-surround organization. This indicates that V4 cells show stereotyped RF properties through selective integration of input activities from cells belonging to a single specific orientation channel. These findings support key properties of our model V4 cells integrating input activations from specific orientation fields generated at previous model stages. The spatial center-surround organization of model V4 cell RF in turn enables the generation of boundary activities at texture discontinuities.

\section{Other neural models of texture processing}

Several computational models exist which do also try to elucidate the neural mechanisms underlying texture segmentation. In many approaches the output energy of a set of filters with different spatial frequency channels (scales) and orientations is computed and further processed for, e.g. response normalization, output combinations from different channels, etc. (Bergen, 1991; Graham et al., 1993; Lee, 1995; Kehrer, 1997; Ontrup et al., unpublished observations). Some of these models incorporate rather sophisticated higher processing stages to demonstrate similar response properties to certain types of textures corresponding with human perception. It still remains a matter of debate whether a bank of differently scaled filters exists in the visual system that is applied to generate a representation of spatial frequency channels from the input luminance signal. For texture segregation, the responses from different frequency representations (and in different orientations) need to be combined, where various mechanisms have been proposed by different authors. Our model differs from these approaches in that cells utilize filters of increasing sizes along the processing pathway in different higher-order model areas. Therefore, the input integration at a particular stage is based on processing results from previous stages and not on the input luminance itself as in multi-scale filtering. Some of these multi-scale models utilize computational strategies based on the detection of borders between different textures (Graham et al., 1993; Kehrer, 1997). They employ a second processing stage containing filters to detect activity changes in the output of the first filter bank, which is to some extent comparable to the computation in our model area V4.

The model perhaps most similar to our V1-V4 feedforward processing stream has been proposed in (Malik and Perona, 1990). In that model, initial V1 responses undergo a non-linear center-surround interaction computed over a neighborhood in the spatial and orientation domain, which is similar to our stage of V1 shunting competition. Furthermore, in that model, these responses are then spatially blurred and gradients are measured in each orientation field separately. Finally, at each spatial location the maximum response is selected over all orientation channels. This cascade of processing stages is a simplified version of the feedforward path in our model V4 computation. Taking the gradient is a computational simplifica- tion of the functionality implemented by our model $\mathrm{V} 4$ cell kernels. The model of Malik and Perona (1990) and our approach have also some parallels with a scheme of texture segregation proposed by Beck (1982). Based on the results of various psychophysical investigations, Beck conjectured the existence of several processing levels involved in texture segregation, namely detection, linking, difference encoding, and (threshold-based) decision for segmentation. Our model incorporates the overall functionality of this proposed hierarchy of processing units. For example, feature detection and linking into boundary fragments is achieved by model cells of areas V1 and V2, respectively. The functionality of difference detectors and decision units is realized by the function of model area V4.

Unlike our approach, the models discussed above are not capable to explain either alignment effects or the possible functional role of feedback from higher visual areas. Unlike the selection of maximal responses in the model of Malik and Perona's (1990) or Beck's (1982) proposal of a hard-coded decision stage, segmentation in our model emerges from V4 center-surround competition generating a map of graded responses and in turn enhancing compatible activity distributions at previous processing stages. The feedback connections enable a recurrent flow of activation, thereby creating more computational flexibility and circumventing simple all-or-none decisions necessary in feed-forward models to generate localized results. The activity pattern in a specific model area is modulated via feedback activity from higher areas in a context-sensitive way. This mechanism enables the overall model to focus processing on the important parts of a visual scene, and thereby gaining robustness against noise and pattern perturbations (compare Figs. 6 and 8b). The key contributions of feedback mechanisms are that they mediate the graded enhancement of boundaries defined by texture OC, together with the suppression of spurious activities that were generated by orientation noise.

Other neural models of boundary processing utilize the initial responses of $\mathrm{V} 1$ simple and complex cells, all having a single, fixed receptive field size, as input for further recurrent processing within and between several model areas. Some of these approaches simulate processes of boundary formation observed in early vision by recurrent interaction of V1 complex cells and V2 bipole cells (Grossberg and Mingolla, 1985; Neumann and Sepp, 1999; Mansson, 2000). These approaches spatially group texture items and can reproduce the high saliency of aligned bars or the induction of illusory contours. The computational capabilities of these models are preserved and incorporated by our approach. In particular, our model shows how grouping of aligned fragments into curved texture flow patterns influences texture boundary detection by model V4 cells (Fig. 7).

In summary, we propose that a variety of visual areas including V1, V2 and V4 is involved in the robust determination of texture boundaries for segmentation of surface regions. It is the assumption of the full hierarchy of areas that permits to construct a biologically plausible model of texture processing. Our approach preserves the capabili- 
ties of recurrent V1-V2 models in contour formation, and results in a model being in accordance with a more general architecture of the parvocellular pathway. In contrast to other models of texture processing, this approach can trace back fundamental psychophysical and electrophysiological results to the likely underlying neural architecture, together with the neural mechanisms of texture border detection, alignment effects and enhanced V1 cell activities at texture borders.

\section{Limitations of the model}

The major goal of our model was to achieve robust detection of borders between oriented textures. The focus on this issue allowed us to employ a model of area V2 which is simplified in comparison with more elaborated models of recurrent V1-V2 processing for contour integration (Grossberg and Mingolla 1985; Neumann and Sepp 1999; see Neumann and Mingolla 2001 for a review of models). These approaches include more sophisticated mechanisms of bipole cell integration, which have better capabilities in completing curved contours. However, the activation patterns of our model area V2 show satisfactory alignment effects. In addition, the restriction to simpler receptive fields permits to reduce the computational complexity while keeping the same computational principles. We modeled area V2 to be exclusively composed of bipole cells. This in turn leads to a very strong influence of alignment effects on the activity pattern observed in model area V4 (see Fig. 7). The dependency of pop-out on alignment effects is in accordance with the psychophysical results presented in (Nothdurft, 1992). Nevertheless, this effect might be less pronounced than predicted by our model. Other cell types in V2, e.g. cells comparable to V1 complex cells which act on a wider spatial scale might attenuate the impact of bipole cells.

\section{CONCLUSION}

The detailed neural mechanisms underlying texture segregation in the human visual system are largely unknown and a matter of ongoing research. In this study, we attempted to decipher these mechanisms by developing a biologically plausible computational model based on anatomical and physiological data. A hierarchical feedback model consisting of bi-directionally connected areas $\mathrm{V} 1$, V2 and V4 each containing a topographic map of orientation selective cells was constructed. Fundamental psychophysical and electrophysiological results indicate that human texture processing is based on the detection of borders defined by salient OCs. Our approach allowed to trace back these observations to the probable neural origins by directly linking them with activation patterns of the computational model. Artificial lesions of specific connections helped to identify the functional role of these connections. In particular, the role of feedback projections between the model areas in the context of texture processing was examined by comparing the full recurrent model with a pure feed-forward network with the full recurrent model and the effects of selectively introduced artificial lesions.
Although our focus was on segmentation of oriented textures, we think that the general ideas of border detection and enhancement via model V4 cells and feedback modulation are not restricted to the orientation domain, but can be extended to detect activity transitions in other fields of pre-processed input. For example, Bergen and Adelson (1988) showed that the responses of cells with simple linear center-surround receptive fields can effectively code the size of texture elements independent of their exact shape. The output of these cells can in turn provide the input to our model of cortical processing, particularly V4 cells, in order to establish borders between texture elements of variable size.

Acknowledgements-We thank the late Jake Beck (Department of Cognitive and Neural Systems, Boston University; deceased in March 2003) for his insightful comments and many helpful suggestions on an earlier version of the manuscript. Georg Grön's (Department of Psychiatry, University of UIm) helpful suggestions are also gratefully acknowledged. The critical comments of the anonymous reviewers helped to improve the manuscript.

\section{REFERENCES}

Beck J (1966) Effect of orientation and of shape similarity on perceptual grouping. Percept Psychophysiol 1:300-302.

Beck J (1982) Textural Segmentation. Organization and representation in perception (In: Beck J, ed), pp 285-317. Hillsdale: Lawrence Erlbaum Associates.

Beck J (1983) Texture segmentation: second-order statistics and textural Elements. Biol Cybern 48:125-130.

Bergen JR (1991) Theories of visual texture perception. In: Spatial vision, Vol. 10 (Regan D, ed), pp 115-134. New York: Macmillan Press.

Bergen JR, Adelson EH (1988) Early vision and texture perception. Nature 333:363-364.

Cavanaugh JR, Bair W, Movshon JA (2002a) Nature and interaction of signals from the receptive field center and surround in macaque $\mathrm{V} 1$ neurons. J Neurophysiol 88:2530-2546.

Cavanaugh JR, Bair W, Movshon JA (2002b) Selectivity and spatial distribution of signals from the receptive field surround in macaque V1 neurons. J Neurophysiol 88:2547-2556.

Crick F, Koch C (1998) Constraints on cortical and thalamic projections: the no-strong-loops hypothesis. Nature 391:245-250.

de Weerd P, Desimone R, Ungerleider LG (1996) Cue-dependent deficits in grating orientation discrimination after V4 lesions in macaques. Vis Neurosci 13:529-538.

Felleman DJ, van Essen DC (1991) Distributed hierarchical processing in the primate cerebral cortex. Cereb Cortex 1:1-47.

Gallant JL, van Essen DC, Nothdurft HC (1995) Two-dimensional and three-dimensional texture processing in visual cortex of the Macaque monkey. In: Early vision and beyond (Papathomas TV, ed), pp 89-98. Cambridge: MIT Press.

Gilbert CD, Wiesel TN (1989) Columnar specificity of intrinisic horizontal and corticocortical connections in cat visual cortex. J Neurosci 9:2432-2442.

Graham N, Sutter A, Venkatesan C (1993) Spatial-frequency- and orientation-selectivity of simple and complex channels in region segregation. Vis Res 33:1893-1911.

Grossberg S (1980) How does a brain build a cognitive code? Psychol Rev 87:1-51.

Grossberg S, Mingolla E (1985) Neural dynamics of perceptual grouping: textures, boundaries, and emergent segmentations. Percept Psychophysiol 38:141-171.

Hansen, T, Neumann, H, (1999) A model of V1 visual contrast pro- 
cessing utilizing long-range connections and recurrent interactions. Proceedings of the ICANN 99, Edinburgh, UK.

Heitger F, van den Heydt R, Peterhans E, Rosenthaler L, Kübler O (1998) Simulation of neural contour mechanisms: representing anomalous contours. Image Vis Comput 6:407-421.

Hirsch JA, Gilbert CD (1991) Synaptic physiology of horizontal connections in the cat's visual cortex. J Neurosci 11:1800-1809.

Hubel DH, Wiesel TN (1962) Receptive fields, binocular interaction and functional architecture in the cat's visual cortex. J Physiol 160:106-154.

Hupé J, James A, Girard P, Bullier J (2001) Response modulations by static texture surround in area $\mathrm{V} 1$ of the macaque monkey do not depend on feedback connections from V2. J Neurophysiol 85:146163.

Hupé JM, James AC, Payne BR, Lomber SG, Girard P, Bullier J (1998) Cortical feedback improves discrimination between figure and background by V1, V2 and V3 neurons. Nature 394:784-787.

Julesz B (1965) Texture and visual perception. Sci Am 212:38-48.

Julesz B (1981) Textons, the elements of texture perception, and their interactions. Nature 290:91-97.

Kapadia MK, Ito M, Gilbert CD, Westheimer G (1995) Improvement in visual sensitivity by changes in local context: parallel studies in human observers and in V1 of alert monkeys. Neuron 15:843-856.

Kapadia MK, Westheimer G, Gilbert CD (2000) Spatial distribution of contextual interactions in primary visual cortex and in visual perception. J Neurophysiol 84:2048-2062.

Kastner S, de Weerd P, Pinsk MA, Elizondo MI, Desimone R, Ungerleider LG (2001) Modulation of sensory suppression: implications for receptive field sizes in the human visual cortex. $J$ Neurophysiol 86:1398-1411.

Kastner S, de Weerd P, Ungerleider LG (2000) Texture segregation in the human visual cortex: a functional MRI study. J Neurophysiol 83:2453-2457.

Kastner S, Ungerleider LG (2000) Mechanisms of visual attention in the human cortex. Annu Rev Neurosci 23:315-341.

Kehrer L (1997) The central performance drop in texture segmentation: a simulation based on a spatial filter model. Biol Cybern 77:297-305.

Lamme V, Rodriguez-Rodriguez V, Spekreijse H (1999) Separate processing dynamics for texture elements, boundaries and surfaces in primary visual cortex of the macaque monkey. Cereb Cortex 9:406-413.

Lamme V, Super H, Spekreijse H (1998) Feedforward, horizontal, and feedback processing in the visual cortex. Curr Opin Neurobiol 8:529-535.

Landy MS, Bergen JR (1991) Texture segregation and orientation gradient. Vision Res 31:679-691.

Lee TS (1995) A bayesian framework for understanding texture segmentation in the primary visual cortex. Vision Res 35:2643-2657.

Li W, Thier P, Wehrhahn C (2000) Contextual influence in orientation discrimination of humans and responses of neurons in $\mathrm{V} 1$ of alert monkeys. J Neurophysiol 83:941-954.

Li W, Thier P, Wehrhahn C (2001) Neural responses from beyond the classic receptive field in V1 of alert monkeys. Exp Brain Res 139: 359-371.

$\mathrm{Li} \mathrm{Z} \mathrm{(1998)} \mathrm{A} \mathrm{neural} \mathrm{model} \mathrm{of} \mathrm{contour} \mathrm{integration} \mathrm{in} \mathrm{the} \mathrm{primary} \mathrm{visual}$ cortex. Neural Comput 10:903-940.

Li Z (2002) A saliency map in primary visual cortex. Trends Cogn Sci 6:9-16.

Malik J, Perona P (1990) Preattentive texture discrimination with early vision mechanisms. J Opt Soc Am A 7:923-932.

Mansson, J (2000) Occluding contours: a computational model of suppressive mechanisms in human contour perception. Proceedings of the Lund University Cognitive Studies, Lund, 81.

Mendola JD, Dale AM, Fischl B, Liu AK, Tootell RBH (1999) The representation of illusory and real contours in human cortical visual areas revealed by functional magnetic resonance imaging. J Neurosci 19:8560-8572.
Merigan WH (1996) Basic visual capacities in shape discrimination after lesions of extrastriate area V4 in macaques. Vis Neurosci 13:51-60.

Merigan WH (2000) Cortical area V4 is critical for certain texture discriminations, but this effect is not dependent on attention. Vis Neurosci 17:949-958.

Mignard M, Malpeli JG (1991) Paths of information flow through visual cortex. Science 251:1249-1251.

Mumford DB (1994) Neuronal architectures for pattern-theoretic problems. In: Large-scale neuronal theories of the brain (Koch C, Davis J, eds), pp 125-152. Cambridge, MA: MIT Press.

Nakamura H, Gattass R, Desimone R, Ungerleider LG (1993) The modular organization of projections from areas $\mathrm{V} 1$ and $\mathrm{V} 2$ to areas $\mathrm{V} 4$ and TEO in macaques. J Neurosci 13:3681-3691.

Neumann H, Mingolla E (2001) Computational neural models of spatial integration in perceptual grouping. In: From fragments to objects: segmentation and grouping in vision (Shipley TF, Kellman PJ, eds), pp 353-400. Amsterdam: Elsevier.

Neumann H, Pessoa L, Hansen T (1999) Interaction of ON and OFF pathways for visual contrast measurement. Biol Cybern 81:515532.

Neumann H, Sepp W (1999) Recurrent V1-V2 interaction in early visual boundary processing. Biol Cybern 81:425-444.

Nothdurft HC (1985) Sensitivity for structure gradient in texture discrimination tasks. Vision Res 25:1957-1968.

Nothdurft HC (1991) Texture segmentation and pop-out from orientation contrast. Vision Res 31:1073-1078.

Nothdurft HC (1992) Feature analysis and the role of similarity in preattentive vision. Percept Psychophysiol 52:355-375.

Nothdurft HC, Gallant JL, van Essen DC (1999) Response modulation by texture surround in primate area $\mathrm{V} 1$ : correlates of "popout" under anesthesia. Vis Neurosci 16:15-34.

Nothdurft HC, Gallant JL, van Essen DC (2000) Response profiles to texture border patterns in V1. Vis Neurosci 17:421-436.

Pasupathy A, Connor CE (2001) Shape representation in area V4: position-specific tuning for boundary conformation. J Neurosci 86 : 2505-2519.

Peterhans E (1997) Functional organization of area V2 in the awake monkey. In: Extrastriate cortex in primates, Vol. 12 (Rockland KS, Kaas JH, Peters A, eds), pp 335-357. New York: Plenum Press.

Pollen DA, Przybyszewski AW, Rubin MA, Foote W (2002) Spatial receptive field organization of macaque $\mathrm{V} 4$ neurons. Cereb Cortex 12:601-616.

Przybyszewski AW, Gaska JP, Foote W, Pollen DA (2000) Striate cortex increases contrast gain of macaque LGN neurons. Vis Neurosci 17:485-494.

Reppas JB, Niyogi S, Dale AM, Sereno MI, Tootell RB (1997) Representation of motion boundaries in retinotopic human visual cortical areas. Nature 388:175-179.

Rockland KS, Saleem KS, Tanaka K (1994) Divergent feedback connections from areas $\mathrm{V} 4$ and TEO in the macaque. Vis Neurosci 11:579-600.

Ross WD, Grossberg S, Mingolla E (2000) Visual cortical mechanisms of perceptual grouping: interacting layers, networks, columns, and maps. Neural Netw 13:571-588.

Salin PA, Bullier J (1995) Corticocortical connections in the visual system: structure and function. Physiol Rev 75:107-154.

Sandell JH, Schiller PH (1982) Effect of cooling area 18 on striate cortex cells in the squirrel monkey. J Neurophysiol 48:38-48.

Sereno MI, Dale AM, Reppas JB, Kwong KK, Belliveau JW, Brady TJ, Rosen BR, Tootell RBH (1995) Borders of multiple visual areas in humans revealed by functional MRI. Science 268:889-893.

Smith AT, Singh KD, Williams AL, Greenlee MW (2001) Estimating receptive field size from $\mathrm{fMRI}$ data in human striate and extrastriate visual cortex. Cereb Cortex 11:1182-1190.

Sporns O, Tononi G, Edelman GM (1991) Modeling perceptual grouping and figure-ground segregation by means of active reentrant connections. Proc Natl Acad Sci USA 88:129-133. 
Tootell RBH, Mendola JD, Hadjikhani NK, Ledden PJ, Liu AK, Reppas JB, Sereno MI, Dale AM (1997) Functional analysis of V3A and related areas in human visual cortex. J Neurosci 17:7060-7078.

Ungerleider LG, Haxby JV (1994) 'What' and 'where' in the human brain. Curr Opin Neurobiol 4:157-165.

von der Heydt R, Heitger F, Peterhans E (1993) Perception of occluding contours: neural mechanisms and a computational model. Biomed Res 14:1-6.

van den Heydt R, Peterhans E, Baumgartner G (1984) Illusory contours and cortical neuron responses. Science 224:1260-1262.

Zilles K, Clarke S (1997) Architecture, connectivity and transmitter receptors of human extrastriate visual cortex: comparison with nonhuman primates. In: Extrastriate cortex in primates, Vol. 12 (Rockland KS, Kaas JH, Peters A, eds), pp. 673-742. New York: Plenum Press.

\section{APPENDIX}

\section{Appendix A: Table 1}

Parameters of the three-stage cell dynamics and S.D. $\sigma$ of the Gaussians pooling the center and surround activity in the spatial and orientational domains (see eq. $1-4)$. The values were kept constant through all experiments.

\section{APPENDIX B}

In the following, the equations describing the receptive field properties are depicted, which determine the initial activation $c$ of a cell. The letter $l^{(2)}$ denotes the final activation of the cells in the prior area after top-down modulation and center-surround competition. Capital letters A, B, C, D or E denote constants. Anisotropic Gaussians in the spatial domain are described by $\Lambda_{\sigma x, \sigma y, \tau x, \tau y, \theta}$. Their size (in pixel) is defined by standard deviations $\sigma_{x}$ and $\sigma_{y}$ in the $x$ - and y-direction, respectively. They are shifted by $\tau_{x}$ and $\tau_{y}$ pixel in the $x$ - and y-direction and finally rotated by angle $\theta$. Spatial locations in the topographical maps are expressed by the index $i$. $\Psi$ denotes isotropic Gaussians in the orientation domain, $*$ is the convolution operator and $[x]^{+}:=\max \{x, 0\}$ stands for half-wave rectification.

\section{LGN ON/OFF cells}

LGN cell activity is determined by convolution of the input image with a difference-of-Gaussians operator followed by a half-wave rectification:

$$
\begin{array}{rll}
x= & l *\left(\Lambda_{\text {Center }}-\Lambda_{\text {Surround }}\right) \\
x^{\text {on }}= & {[\mathrm{x}]^{+}} \\
x^{\text {off }}= & {[-\mathrm{x}]^{+}} \\
\sigma_{\text {Center }}=0.8 ; & & \sigma_{\text {Surround }}=3 \sigma_{\text {Center }}
\end{array}
$$

\section{V1 simple cells}

Simple cells have elongated subfields which pool the input of appropriately aligned LGN responses. The subfields are shifted perpendicular to their main axis and then rotated by $\theta$. The activity of the subfields denoted by $p$ is subsequently fed into a softAND-circuit to determine the response $s$ of the simple cell. Simple cells exist for two polarities (dark-light, dl; light-dark, Id) and eight orientations.

The activation $s_{i \theta}^{l d}$ of a simple cell sensitive for polarity light-dark is given by:

$s_{i \theta}^{\text {Id }}=\frac{A_{s}\left(p_{i \theta}^{\text {on } \_ \text {left }}+p_{i \theta}^{\text {off_right }}\right)+2 B_{s} p_{i \theta}^{\text {on } \_ \text {left }} p_{i \theta}^{\text {off_right }}}{A_{s} D_{s}+E_{s}\left(p_{i \theta}^{\text {on } \_ \text {left }}+p_{i \theta}^{\text {off }}{ }^{\text {ight }}\right)}$

with:

$$
\begin{aligned}
& p_{\theta}^{\text {on } \_ \text {left }}=x^{\text {on }} * \Lambda_{\sigma x, \sigma y, 0,-\tau_{y} / 2, \theta} \\
& p_{\theta}^{\text {off_right }}=x^{\text {off } *} \Lambda_{\sigma x, \sigma y, 0, \tau_{y} / 2, \theta} \\
& p_{\theta}^{\text {off_left }}=\ldots ; p_{\theta}^{\text {on_right }}=\ldots \\
& s_{i \theta}{ }^{d l}=\ldots \\
& \sigma_{y}=0.8 ; \sigma_{x}=3.5 \sigma_{y} ; \tau_{y}=1.6 \sigma_{y} \\
& A_{s}=1.0 ; B_{s}=10000.0 ; D_{s}=0.05 ; E_{\mathrm{s}}=100.0 \\
& n_{\text {orient }}=8 ; \theta=0, \pi / n_{\text {orient }}, \ldots,\left(n_{\text {orient }}-1\right) \pi / n_{\text {orient }}
\end{aligned}
$$

\section{V1 complex cells}

The initial $\mathrm{V} 1$ complex cell response $c_{i \theta}$ is determined by

\begin{tabular}{|c|c|c|c|c|c|c|c|c|c|c|c|c|}
\hline & \multicolumn{4}{|c|}{ Parameters for $I^{(1)}$} & \multicolumn{8}{|c|}{ Parameters for $l^{(2)}$} \\
\hline & \multirow[t]{2}{*}{$\alpha_{1}$} & \multirow[t]{2}{*}{$\beta_{1}$} & \multirow[t]{2}{*}{$\gamma_{1}$} & \multirow[t]{2}{*}{ C } & \multirow[t]{2}{*}{$\alpha_{2}$} & \multirow[t]{2}{*}{$\beta_{2}$} & \multirow[t]{2}{*}{$\delta_{2}$} & \multirow[t]{2}{*}{$\zeta_{2}$} & \multicolumn{4}{|l|}{$\sigma$ of } \\
\hline & & & & & & & & & $\Psi^{+}$ & $\Lambda^{+}$ & $\Psi^{-}$ & $\Lambda^{-}$ \\
\hline V1 & 12.0 & 0.73 & 3.7 & 25 & 1.0 & 2.8 & 3.5 & 5.0 & 0.2 & 1.0 & 2.0 & 3.0 \\
\hline V2 & 12.0 & 0.85 & 4.2 & 20 & 1.0 & 2.9 & 3.1 & 50.0 & 0.2 & 2.0 & 2.0 & 6.0 \\
\hline V4 & - & - & - & - & 1.0 & 10.6 & 9.9 & 1000 & 0.2 & 8.0 & 2.0 & 24.0 \\
\hline
\end{tabular}
the sum of half-wave rectified differences of the two simple cell activities of opposite polarity (dark-light and lightdark) at each position:

$$
\begin{aligned}
& c_{i \theta}=A_{c}\left(\left[S_{i \theta}^{d d}-S_{i \theta}^{d l}\right]^{+}+\left[S_{i \theta}^{d l}-S_{i \theta}^{d d}\right]^{+}\right) \\
& A_{c}=0.1
\end{aligned}
$$

\section{V2 bipole cells}

Model bipole cells consist of two prolated subfields which are shifted parallel to the main axis of the cell. They pool

Table 1. Parameters of the three-stage cell dynamics and S.D. $\sigma$ of the Gaussians pooling the center and surround activity in the spatial and orientational domains (see eq. 1-4) 
the activity $I_{V 1}^{(2)}$ of $\mathrm{V} 1$ complex cells, which are approximately sensitive to the same orientation as the bipole cell. The activities $f$ of the subfields are combined using a soft-ANDgate to determine the initial activation $c_{i \theta}$ of the cell. The subfields $K$ are modeled as anisotropic Gaussians, which are cut off in the central part of the cell by means of a sigmoid function. The partial overlap of the subfields in the center of the cell defines the classical receptive field.

The initial activation $c_{i \theta}$ of a V2 bipole cell is given by:

$c_{i \theta}=\frac{A_{t}\left(f_{i \theta}^{\text {left }}+f_{i \theta}^{\text {right }}\right)+2 B_{t} f_{i \theta}^{\text {left }} f_{i \theta}^{\text {right }}}{A_{t} D_{t}+E_{t}\left(f_{i \theta}^{\text {left }}+f_{i \theta}^{\text {right }}\right) .}$

with:

$$
\begin{aligned}
f^{\text {left }} & =I_{V 1}^{(2)} * \Psi_{f} * K^{\text {left }} \\
f^{\text {right }} & =l_{V 1}^{(2)} * \Psi_{f} * K^{\text {right }}
\end{aligned}
$$

Subfields:

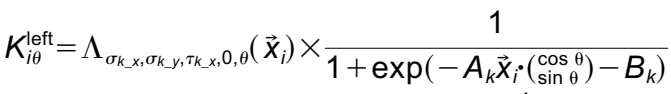

$$
\begin{aligned}
& K_{i \theta}^{\text {right }}=\Lambda_{\sigma_{k_{-}, x}, \sigma_{k_{-},},-\tau_{k_{-}, x, \theta}, \theta}\left(\vec{x}_{i}\right) \times \frac{1}{1+\exp \left(+A_{k} \vec{x}_{i} \cdot\left(\begin{array}{c}
\sin \theta \\
\cos \theta
\end{array}\right)+B_{k}\right)}
\end{aligned}
$$

$\vec{X}_{i}$ : cartesian coordinates of point $i$

$$
\begin{aligned}
& \sigma_{k \_x}=22.0 ; \sigma_{k \_y}=1.0 ; t_{k \_x}=2.0 ; \mathrm{A}_{\mathrm{k}}=0.8 ; \mathrm{B}_{\mathrm{k}}=9.0 \\
& \mathrm{~A}_{\mathrm{t}}=2.3 ; \mathrm{B}_{\mathrm{t}}=2600.0 ; \mathrm{D}_{\mathrm{t}}=0.15 ; \mathrm{E}_{\mathrm{t}}=100.0 \\
& \psi_{f}: \sigma_{f_{-} \text {orient }}=0.3
\end{aligned}
$$

\section{Model V4 cells}

Model V4 cells consist of one central excitatory and two lateral inhibitory elongated subfields all sensitive to the same input orientation. The lateral inhibitory fields are shifted perpendicularly to their main axis. The differences between the $\mathrm{V} 2$ bipole cell activities $l_{V 2}^{(2)}$ pooled by the center field and the left and right inhibitory subfields are determined. The sum of the two half-wave rectified differences is the initial activation of the V4 cell. For each of the eight $\mathrm{V} 1$ complex cell orientations (denoted by the angle $\theta), \mathrm{V} 4$ cells in eight orientations exist (denoted by the angle $\varphi)$.

The initial activation $\mathrm{c}_{i \theta \varphi}$ of a model V4 cell is given by:

$$
c_{i \theta \phi}=\left[q_{i \theta \phi}^{\text {center }}-C q_{i \theta \phi}^{\text {left }}\right]^{+}+\left[q_{i \theta \phi}^{\text {center }}-C q_{i \theta \phi}^{\text {right }}\right]^{+}
$$

with:

$$
\begin{aligned}
& q_{\phi}^{\text {center }}=l_{V 2}^{(2)} * \Psi_{q} * \Lambda_{\sigma_{q_{-}, \sigma_{q}, 0,0, \phi}} \\
& q_{\phi}^{\text {left }}=l_{V 2}^{(2)} * \Psi_{q} * \Lambda_{\sigma_{q_{-}, \sigma_{q}-}, 0,-\tau_{q_{-}, \phi}} \\
& q_{\phi}^{\text {right }}=l_{V 2}^{(2)} * \Psi_{q} * \Lambda_{\sigma_{q_{-}, \sigma_{q_{-},}, 0, \tau_{q_{-}, \phi}, \phi}} \\
& \sigma_{q_{-} x}=22.0 ; \sigma_{q_{-} y}=8.0 ; \tau_{q_{-} y}=16.0 ; C=1.25 \\
& \psi_{q}: \sigma_{q \_ \text {orient } \_\theta}=0.4 \\
& \varphi=0, \pi / n_{\text {orient }}, \ldots,\left(n_{\text {orient }}-1\right) \pi / n_{\text {orient }}
\end{aligned}
$$

As V4 constitutes the highest model area, the initial activation $c_{i \theta \varphi}$ is not modulated via feedback, but is directly fed into the center-surround competition denoted by equation (3). In this competition, all V4 cells sensitive to the same V1 orientation interact in a spatial and orientational (denoted by $\varphi$ ) neighborhood. No interaction between different V1 orientation channels exists in model area V4. The final responses $I_{V 4}^{(2)}$ of all V4 cells sensitive to the same $\mathrm{V} 1$ orientation are summed up after center-surround competition and fed back to V2:

$I_{V 4 i \theta}^{(2)}=\sum_{k=1}^{n_{\text {orient }}} I_{V 4 i \theta(k-1) \times \pi / n_{\text {orient }}}^{(2)}$ 